\title{
Measurement of the Branching Fractions for the Exclusive Decays of $B^{0}$ and $B^{+}$to $\bar{D}^{(*)} D^{(*)} K$
}

\author{
B. Aubert, R. Barate, D. Boutigny, J.-M. Gaillard, A. Hicheur, \\ Y. Karyotakis, J. P. Lees, P. Robbe, V. Tisserand, and A. Zghiche \\ Laboratoire de Physique des Particules, F-74941 Annecy-le-Vieux, France \\ A. Palano and A. Pompili \\ Università di Bari, Dipartimento di Fisica and INFN, I-70126 Bari, Italy \\ J. C. Chen, N. D. Qi, G. Rong, P. Wang, and Y. S. Zhu \\ Institute of High Energy Physics, Beijing 100039, China \\ G. Eigen, I. Ofte, and B. Stugu \\ University of Bergen, Inst. of Physics, N-5007 Bergen, Norway
}

G. S. Abrams, A. W. Borgland, A. B. Breon, D. N. Brown, J. Button-Shafer, R. N. Cahn, E. Charles, C. T. Day, M. S. Gill, A. V. Gritsan, Y. Groysman, R. G. Jacobsen, R. W. Kadel, J. Kadyk, L. T. Kerth, Yu. G. Kolomensky, J. F. Kral, G. Kukartsev, C. LeClerc, M. E. Levi, G. Lynch, L. M. Mir, P. J. Oddone, T. J. Orimoto, M. Pripstein, N. A. Roe, A. Romosan, M. T. Ronan, V. G. Shelkov, A. V. Telnov, and W. A. Wenzel Lawrence Berkeley National Laboratory and University of California, Berkeley, CA 94720, USA
T. J. Harrison, C. M. Hawkes, D. J. Knowles, R. C. Penny, A. T. Watson, and N. K. Watson University of Birmingham, Birmingham, B15 2TT, United Kingdom

T. Deppermann, K. Goetzen, H. Koch, B. Lewandowski, M. Pelizaeus, K. Peters, H. Schmuecker, and M. Steinke Ruhr Universität Bochum, Institut für Experimentalphysik 1, D-44780 Bochum, Germany

N. R. Barlow, J. T. Boyd, N. Chevalier, W. N. Cottingham, C. Mackay, and F. F. Wilson University of Bristol, Bristol BS8 1TL, United Kingdom

C. Hearty, T. S. Mattison, J. A. McKenna, and D. Thiessen University of British Columbia, Vancouver, BC, Canada V6T 1 Z1

P. Kyberd and A. K. McKemey Brunel University, Uxbridge, Middlesex UB8 3PH, United Kingdom

V. E. Blinov, A. D. Bukin, V. B. Golubev, V. N. Ivanchenko, E. A. Kravchenko, A. P. Onuchin, S. I. Serednyakov, Yu. I. Skovpen, E. P. Solodov, and A. N. Yushkov Budker Institute of Nuclear Physics, Novosibirsk 630090, Russia

D. Best, M. Chao, D. Kirkby, A. J. Lankford, M. Mandelkern, S. McMahon, R. K. Mommsen, W. Roethel, and D. P. Stoker University of California at Irvine, Irvine, CA 92697, USA

\author{
C. Buchanan \\ University of California at Los Angeles, Los Angeles, CA 90024, USA
}

D. del Re, H. K. Hadavand, E. J. Hill, D. B. MacFarlane, H. P. Paar, Sh. Rahatlou, U. Schwanke, and V. Sharma University of California at San Diego, La Jolla, CA 92093, USA 
J. W. Berryhill, C. Campagnari, B. Dahmes, N. Kuznetsova, S. L. Levy, O. Long, A. Lu, M. A. Mazur, J. D. Richman, and W. Verkerke University of California at Santa Barbara, Santa Barbara, CA 93106, USA

J. Beringer, A. M. Eisner, C. A. Heusch, W. S. Lockman, T. Schalk, R. E. Schmitz, B. A. Schumm, A. Seiden, M. Turri, W. Walkowiak, D. C. Williams, and M. G. Wilson University of California at Santa Cruz, Institute for Particle Physics, Santa Cruz, CA 95064, USA

J. Albert, E. Chen, M. P. Dorsten, G. P. Dubois-Felsmann, A. Dvoretskii,

D. G. Hitlin, I. Narsky, F. C. Porter, A. Ryd, A. Samuel, and S. Yang California Institute of Technology, Pasadena, CA 91125, USA

S. Jayatilleke, G. Mancinelli, B. T. Meadows, and M. D. Sokoloff University of Cincinnati, Cincinnati, OH 45221, USA

T. Abe, T. Barillari, F. Blanc, P. Bloom, P. J. Clark, W. T. Ford, U. Nauenberg, A. Olivas, P. Rankin, J. Roy, J. G. Smith, W. C. van Hoek, and L. Zhang University of Colorado, Boulder, CO 80309, USA

J. L. Harton, T. Hu, A. Soffer, W. H. Toki, R. J. Wilson, and J. Zhang Colorado State University, Fort Collins, CO 80523, USA

D. Altenburg, T. Brandt, J. Brose, T. Colberg, M. Dickopp, R. S. Dubitzky, A. Hauke, H. M. Lacker, E. Maly,

R. Müller-Pfefferkorn, R. Nogowski, S. Otto, K. R. Schubert, R. Schwierz, B. Spaan, and L. Wilden

Technische Universität Dresden, Institut für Kern- und Teilchenphysik, D-01062 Dresden, Germany

D. Bernard, G. R. Bonneaud, F. Brochard, J. Cohen-Tanugi, Ch. Thiebaux, G. Vasileiadis, and M. Verderi Ecole Polytechnique, LLR, F-91128 Palaiseau, France

A. Khan, D. Lavin, F. Muheim, S. Playfer, J. E. Swain, and J. Tinslay University of Edinburgh, Edinburgh EH9 3JZ, United Kingdom

D. Bettoni, C. Bozzi, R. Calabrese, L. Piemontese, and A. Sarti Università di Ferrara, Dipartimento di Fisica and INFN, I-44100 Ferrara, Italy

E. Treadwell

Florida A\&M University, Tallahassee, FL 3230\%, USA

F. Anulli,* R. Baldini-Ferroli, M. E. Biagini, A. Calcaterra, R. de Sangro, D. Falciai, G. Finocchiaro, P. Patteri, I. M. Peruzzi, ${ }^{*}$ M. Piccolo, and A. Zallo Laboratori Nazionali di Frascati dell'INFN, I-00044 Frascati, Italy

A. Buzzo, R. Contri, G. Crosetti, M. Lo Vetere, M. Macri, M. R. Monge, S. Passaggio, F. C. Pastore, C. Patrignani, E. Robutti, A. Santroni, and S. Tosi Università di Genova, Dipartimento di Fisica and INFN, I-16146 Genova, Italy

S. Bailey and M. Morii

Harvard University, Cambridge, MA 02138, USA

G. J. Grenier, S.-J. Lee, and U. Mallik

University of Iowa, Iowa City, IA 52242, USA

J. Cochran, H. B. Crawley, J. Lamsa, W. T. Meyer, S. Prell, E. I. Rosenberg, and J. Yi Iowa State University, Ames, IA 50011-3160, USA

M. Davier, G. Grosdidier, A. Höcker, S. Laplace, F. Le Diberder, V. Lepeltier, A. M. Lutz, T. C. Petersen, S. Plaszczynski, M. H. Schune, L. Tantot, and G. Wormser 
Laboratoire de l'Accélérateur Linéaire, F-91898 Orsay, France

R. M. Bionta, V. Brigljević, C. H. Cheng, D. J. Lange, and D. M. Wright Lawrence Livermore National Laboratory, Livermore, CA 94550, USA

A. J. Bevan, J. R. Fry, E. Gabathuler, R. Gamet, M. Kay, D. J. Payne, R. J. Sloane, and C. Touramanis University of Liverpool, Liverpool L69 3BX, United Kingdom

M. L. Aspinwall, W. Bhimji, D. A. Bowerman, P. D. Dauncey, U. Egede, I. Eschrich, G. W. Morton, J. A. Nash, P. Sanders, and G. P. Taylor University of London, Imperial College, London, SW7 2BW, United Kingdom

J. J. Back, P. F. Harrison, H. W. Shorthouse, P. Strother, and P. B. Vidal Queen Mary, University of London, E1 4NS, United Kingdom

G. Cowan, H. U. Flaecher, S. George, M. G. Green, A. Kurup, C. E. Marker,

T. R. McMahon, S. Ricciardi, F. Salvatore, G. Vaitsas, and M. A. Winter

University of London, Royal Holloway and Bedford New College, Egham, Surrey TW20 OEX, United Kingdom

D. Brown and C. L. Davis

University of Louisville, Louisville, KY 40292, USA

J. Allison, R. J. Barlow, A. C. Forti, P. A. Hart, F. Jackson,

G. D. Lafferty, A. J. Lyon, J. H. Weatherall, and J. C. Williams

University of Manchester, Manchester M13 9PL, United Kingdom

A. Farbin, A. Jawahery, D. Kovalskyi, C. K. Lae, V. Lillard, and D. A. Roberts University of Maryland, College Park, MD 20742, USA

G. Blaylock, C. Dallapiccola, K. T. Flood, S. S. Hertzbach, R. Kofler,

V. B. Koptchev, T. B. Moore, S. Saremi, H. Staengle, and S. Willocq

University of Massachusetts, Amherst, MA 01003, USA

R. Cowan, G. Sciolla, F. Taylor, and R. K. Yamamoto

Massachusetts Institute of Technology, Laboratory for Nuclear Science, Cambridge, MA 02139, USA

D. J. J. Mangeol, M. Milek, and P. M. Patel

McGill University, Montréal, QC, Canada H3A $2 T 8$

A. Lazzaro and F. Palombo

Università di Milano, Dipartimento di Fisica and INFN, I-20133 Milano, Italy

J. M. Bauer, L. Cremaldi, V. Eschenburg, R. Godang, R. Kroeger,

J. Reidy, D. A. Sanders, D. J. Summers, and H. W. Zhao

University of Mississippi, University, MS 38677, USA

C. Hast and P. Taras

Université de Montréal, Laboratoire René J. A. Lévesque, Montréal, QC, Canada H3C 3J7

H. Nicholson

Mount Holyoke College, South Hadley, MA 01075, USA

C. Cartaro, N. Cavallo, G. De Nardo, F. Fabozzi, ${ }^{\dagger}$ C. Gatto, L. Lista, P. Paolucci, D. Piccolo, and C. Sciacca Università di Napoli Federico II, Dipartimento di Scienze Fisiche and INFN, I-80126, Napoli, Italy

M. A. Baak and G. Raven

NIKHEF, National Institute for Nuclear Physics and High Energy Physics, 1009 DB Amsterdam, The Netherlands 
J. M. LoSecco

University of Notre Dame, Notre Dame, IN 46556, USA

T. A. Gabriel

Oak Ridge National Laboratory, Oak Ridge, TN 37831, USA

B. Brau and T. Pulliam

Ohio State University, Columbus, OH 43210, USA

J. Brau, R. Frey, M. Iwasaki, C. T. Potter, N. B. Sinev, D. Strom, and E. Torrence University of Oregon, Eugene, OR 97403, USA

F. Colecchia, A. Dorigo, F. Galeazzi, M. Margoni, M. Morandin, M. Posocco, M. Rotondo, F. Simonetto, R. Stroili, G. Tiozzo, and C. Voci Università di Padova, Dipartimento di Fisica and INFN, I-35131 Padova, Italy

M. Benayoun, H. Briand, J. Chauveau, P. David, Ch. de la Vaissière, L. Del Buono, O. Hamon, Ph. Leruste, J. Malcles, J. Ocariz, M. Pivk, L. Roos, J. Stark, and S. T'Jampens Universités Paris VI et VII, Lab de Physique Nucléaire H. E., F-75252 Paris, France

P. F. Manfredi and V. Re Università di Pavia, Dipartimento di Elettronica and INFN, I-27100 Pavia, Italy

L. Gladney, Q. H. Guo, and J. Panetta University of Pennsylvania, Philadelphia, PA 19104, USA

C. Angelini, G. Batignani, S. Bettarini, M. Bondioli, F. Bucci, G. Calderini, M. Carpinelli, F. Forti, M. A. Giorgi, A. Lusiani, G. Marchiori, F. Martinez-Vidal,,$\ddagger$ M. Morganti, N. Neri, E. Paoloni, M. Rama, G. Rizzo, F. Sandrelli, and J. Walsh Università di Pisa, Dipartimento di Fisica, Scuola Normale Superiore and INFN, I-56127 Pisa, Italy

M. Haire, D. Judd, K. Paick, and D. E. Wagoner Prairie View Aछ̈M University, Prairie View, TX 77446, USA

N. Danielson, P. Elmer, C. Lu, V. Miftakov, J. Olsen, A. J. S. Smith, and E. W. Varnes Princeton University, Princeton, NJ 08544, USA

F. Bellini, F. Ferrarotto, F. Ferroni, M. Gaspero, E. Leonardi, M. A. Mazzoni, S. Morganti, M. Pierini, G. Piredda, F. Safai Tehrani, M. Serra, and C. Voena Università di Roma La Sapienza, Dipartimento di Fisica and INFN, I-00185 Roma, Italy

\section{G. Cavoto}

Princeton University, Princeton, NJ 08544, USA and

Università di Roma La Sapienza, Dipartimento di Fisica and INFN, I-00185 Roma, Italy

R. Faccini

University of California at San Diego, La Jolla, CA 92093, USA and

Università di Roma La Sapienza, Dipartimento di Fisica and INFN, I-00185 Roma, Italy

S. Christ, G. Wagner, and R. Waldi

Universität Rostock, D-18051 Rostock, Germany

T. Adye, N. De Groot, B. Franek, N. I. Geddes, G. P. Gopal, E. O. Olaiya, and S. M. Xella Rutherford Appleton Laboratory, Chilton, Didcot, Oxon, OX11 OQX, United Kingdom

R. Aleksan, S. Emery, A. Gaidot, S. F. Ganzhur, P.-F. Giraud, G. Hamel de Monchenault, W. Kozanecki, M. Langer, G. W. London, B. Mayer, G. Schott, G. Vasseur, Ch. Yeche, and M. Zito DAPNIA, Commissariat à l'Energie Atomique/Saclay, F-91191 Gif-sur-Yvette, France 
M. V. Purohit, A. W. Weidemann, and F. X. Yumiceva

University of South Carolina, Columbia, SC 29208, USA

D. Aston, R. Bartoldus, N. Berger, A. M. Boyarski, O. L. Buchmueller, M. R. Convery, D. P. Coupal, D. Dong, J. Dorfan, D. Dujmic, W. Dunwoodie, R. C. Field, T. Glanzman, S. J. Gowdy, E. Grauges-Pous, T. Hadig, V. Halyo, T. Hryn'ova, W. R. Innes, C. P. Jessop, M. H. Kelsey, P. Kim, M. L. Kocian, U. Langenegger,

D. W. G. S. Leith, S. Luitz, V. Luth, H. L. Lynch, H. Marsiske, S. Menke, R. Messner, D. R. Muller, C. P. O'Grady, V. E. Ozcan, A. Perazzo, M. Perl, S. Petrak, B. N. Ratcliff, S. H. Robertson, A. Roodman, A. A. Salnikov, R. H. Schindler, J. Schwiening, G. Simi, A. Snyder, A. Soha, J. Stelzer, D. Su, M. K. Sullivan, H. A. Tanaka, J. Va'vra, S. R. Wagner, M. Weaver, A. J. R. Weinstein, W. J. Wisniewski, D. H. Wright, and C. C. Young Stanford Linear Accelerator Center, Stanford, CA 94309, USA

P. R. Burchat, T. I. Meyer, and C. Roat Stanford University, Stanford, CA 94305-4060, USA

S. Ahmed, M. S. Alam, J. A. Ernst, and F. R. Wappler State Univ. of New York, Albany, NY 12222, USA

W. Bugg, M. Krishnamurthy, and S. M. Spanier University of Tennessee, Knoxville, TN 37996, USA

R. Eckmann, H. Kim, J. L. Ritchie, and R. F. Schwitters University of Texas at Austin, Austin, TX 78712, USA

J. M. Izen, I. Kitayama, X. C. Lou, and S. Ye University of Texas at Dallas, Richardson, TX 75083, USA

F. Bianchi, M. Bona, F. Gallo, and D. Gamba Università di Torino, Dipartimento di Fisica Sperimentale and INFN, I-10125 Torino, Italy

C. Borean, L. Bosisio, G. Della Ricca, S. Dittongo, S. Grancagnolo, L. Lanceri, P. Poropat, ${ }^{\S}$ L. Vitale, and G. Vuagnin Università di Trieste, Dipartimento di Fisica and INFN, I-34127 Trieste, Italy

R. S. Panvini

Vanderbilt University, Nashville, TN 37235, USA

Sw. Banerjee, C. M. Brown, D. Fortin, P. D. Jackson, R. Kowalewski, and J. M. Roney University of Victoria, Victoria, BC, Canada V8W $3 P 6$

H. R. Band, S. Dasu, M. Datta, A. M. Eichenbaum, H. Hu, J. R. Johnson, P. E. Kutter, H. Li, R. Liu, F. Di Lodovico, A. Mihalyi, A. K. Mohapatra, Y. Pan, R. Prepost, S. J. Sekula, J. H. von Wimmersperg-Toeller, J. Wu, S. L. Wu, and Z. Yu University of Wisconsin, Madison, WI 53706, USA

\section{H. Neal}

Yale University, New Haven, CT 06511, USA

(Dated: May 5, 2003)

\section{Abstract}

We report the observation of $823 \pm 57 B^{0}$ and $970 \pm 65 B^{+}$decays to doubly charmed final states $\bar{D}^{(*)} D^{(*)} K$, where $\bar{D}^{(*)}$ and $D^{(*)}$ are fully reconstructed and $K$ is either a $K^{ \pm}$or a $K^{0}$. We use a sample of $82.3 \pm 0.9$ million $B \bar{B}$ events collected between 1999 and 2002 with the BABAR detector at the PEP-II storage ring at the Stanford Linear Accelerator Center. The 22 possible $B$ decays to $\bar{D}^{(*)} D^{(*)} K$ are reconstructed exclusively and the corresponding branching fractions or limits are determined. The branching fractions of the $B^{0}$ and of the $B^{+}$to $\bar{D}^{(*)} D^{(*)} K$ are found to be

$$
\begin{aligned}
\mathcal{B}\left(B^{0} \rightarrow \bar{D}^{(*)} D^{(*)} K\right) & =(4.3 \pm 0.3 \text { (stat) } \pm 0.6 \text { (syst })) \%, \\
\mathcal{B}\left(B^{+} \rightarrow \bar{D}^{(*)} D^{(*)} K\right) & =(3.5 \pm 0.3(\text { stat }) \pm 0.5(\text { syst })) \% .
\end{aligned}
$$


A search for decays to orbitally excited $D_{s}$ states, $B \rightarrow \bar{D}^{(*)} D_{s J}^{+}\left(D_{s J}^{+} \rightarrow D^{(*) 0} K^{+}\right)$is also performed.

PACS numbers: 13.25.Hw, 12.15.Hh, 11.30.Er

\section{INTRODUCTION}

The inconsistency between the measured $b \rightarrow c \bar{c} s$ rate and the rate of semileptonic $B$ decays has been a longstanding problem in $B$ physics. Until 1994, it was believed that the $b \rightarrow c \bar{c} s$ transition was dominated by decays $B \rightarrow D_{s} X$, with some smaller contributions from decays to charmonium states and to charmed strange baryons. Therefore, the $b \rightarrow c \bar{c} s$ branching fraction was computed from the inclusive $B \rightarrow D_{s} X, B \rightarrow(c \bar{c}) X$, and $B \rightarrow \Xi_{c} X$ branching fractions, leading to $\mathcal{B}(b \rightarrow$ $c \bar{c} s)=(15.8 \pm 2.8) \%$ [1]. Theoretical calculations are unable to simultaneously describe this low branching fraction and the semileptonic branching fraction of the $B$ meson [2].

As a possible explanation of this problem, it has been conjectured [3] that $\mathcal{B}(b \rightarrow c \bar{c} s)$ is larger and that decays of the type $B \rightarrow \bar{D}^{(*)} D^{(*)} K(X)$ (where $D^{(*)}$ can be either a $D^{0}, D^{* 0}, D^{+}$, or $\left.D^{*+}\right)$ could contribute significantly to the decay rate. This might also include possible decays to orbitally-excited $D_{s}$ mesons, $B \rightarrow \bar{D}^{(*)} D_{s J}$, followed by $D_{s J} \rightarrow D^{(*)} K$. Experimental evidence in support of this picture has been published in the past few years. This evidence includes the measured branching fraction for wrong-sign $D$ production, averaged over charged and neutral $B$ mesons, by CLEO [4] $[\mathcal{B}(B \rightarrow$ $D X)=(7.9 \pm 2.2) \%]$, and the observation of a small number of fully reconstructed decays $B \rightarrow \bar{D}^{(*)} D^{(*)} K$, both by CLEO [5] and ALEPH [6]. More recently, BABAR [7] and Belle [8] have reported some preliminary results on the evidence for transitions $B^{0} \rightarrow D^{*-} D^{(*) 0} K^{+}$with much larger data sets.

$B \rightarrow \bar{D}^{(*)} D^{(*)} K$ decays can proceed through two different amplitudes: external $W$-emission amplitudes and internal $W$-emission amplitudes (also called colorsuppressed amplitudes). Some decay modes proceed purely through one of these amplitudes while others can proceed through both. Figure 1 shows the possible types for $B \rightarrow \bar{D}^{(*)} D^{(*)} K$ decays. In $B A B A R$, the large data sets now available allow comprehensive investigations of these transitions. In this paper, we present measurements of or limits on the branching fractions for all the possible $B \rightarrow \bar{D}^{(*)} D^{(*)} K^{0}$ and $B \rightarrow \bar{D}^{(*)} D^{(*)} K^{+}$decay modes, using events in which both $D$ mesons are fully reconstructed. Charge conjugate reactions are assumed

\footnotetext{
*Also with Università di Perugia, Perugia, Italy

†Also with Università della Basilicata, Potenza, Italy

¥Also with IFIC, Instituto de Física Corpuscular, CSICUniversidad de Valencia, Valencia, Spain

$\S$ Deceased
}

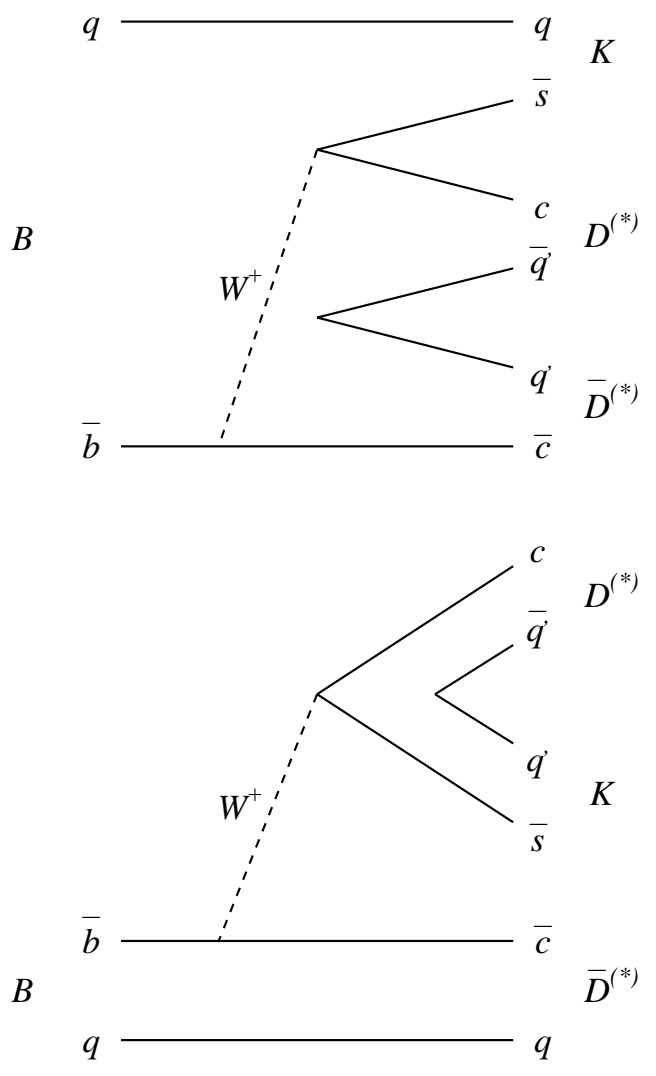

FIG. 1: Top: internal $W$-emission diagram for the decays $B \rightarrow \bar{D}^{(*)} D^{(*)} K$. Bottom: external $W$-emission diagram for the decays $B \rightarrow \bar{D}^{(*)} D^{(*)} K$.

throughout this paper and branching fractions are averaged accordingly.

\section{THE BABAR DETECTOR AND DATASET}

The study reported here uses $75.9 \mathrm{fb}^{-1}$ of data collected at the $\Upsilon(4 S)$ resonance with the BABAR detector at the PEP-II asymmetric-energy $B$ factory, corresponding to $(82.3 \pm 0.9) \times 10^{6} B \bar{B}$ pairs.

The $B A B A R$ detector is a large-acceptance solenoidal spectrometer $(1.5 \mathrm{~T})$ described in detail elsewhere [9]. The analysis described below makes use of charged track and $\pi^{0}$ reconstruction and charged particle identification. Charged particle trajectories are measured by a 5 -layer double-sided silicon vertex tracker (SVT) and a 40-layer drift chamber $(\mathrm{DCH})$, which also provide ionization measurements $(\mathrm{d} E / \mathrm{d} x)$ used for particle identification. For 
charged tracks with $p>1 \mathrm{GeV} / c$, the measured transverse momentum with respect to the beam axis $\left(p_{T}\right)$ has a resolution $\sigma_{p_{T}}$ such that

$$
\frac{\sigma_{p_{T}}}{p_{T}}=0.13 \% p_{T}+0.45 \%,
$$

where $p_{T}$ is measured in $\mathrm{GeV} / c$.

Photons and electrons are measured in the electromagnetic calorimeter (EMC). The EMC consists of 6580 Thallium-doped CsI crystals, which instrument the barrel and forward endcap; the crystals are arranged in a quasi-projective geometry. The electromagnetic calorimeter resolution $\sigma_{E}$ can be expressed as

$$
\frac{\sigma_{E}}{E}=\frac{2.3 \%}{E^{\frac{1}{4}}} \oplus 1.9 \%,
$$

where the energy $E$ is measured in $\mathrm{GeV}$.

Charged particle identification is provided by the average energy loss $(\mathrm{d} E / \mathrm{d} x)$ in the tracking devices and by an internally reflecting ring-imaging Cherenkov detector (DIRC). The DIRC comprises 144 quartz bars, divided into 12 sectors, which transport the Cherenkov light to a water-filled expansion volume equipped with 10751 photomultiplier tubes. A $K / \pi$ separation better than four standard deviations is achieved for momenta below $3 \mathrm{GeV} / c$.

\section{III. $B$ CANDIDATE SELECTION}

The $B^{0}$ and $B^{+}$mesons are reconstructed in a sample of hadronic events for all the possible $\bar{D} D K$ modes, namely $B^{0} \rightarrow D^{(*)-} D^{(*) 0} K^{+}, D^{(*)-} D^{(*)+} K^{0}$, $\bar{D}^{(*) 0} D^{(*) 0} K^{0}$ and $B^{+} \rightarrow \bar{D}^{(*) 0} D^{(*)+} K^{0}, \bar{D}^{(*) 0} D^{(*) 0} K^{+}$, $D^{(*)-} D^{(*)+} K^{+} . K^{0}$ mesons are reconstructed only from the decays $K_{S}^{0} \rightarrow \pi^{+} \pi^{-}$. To eliminate the background from continuum $e^{+} e^{-} \rightarrow q \bar{q}$ events, we require that the ratio of the second to zeroth Fox-Wolfram moments of the event [10] be less than 0.45 .

The $K_{S}^{0}$ candidates are reconstructed from two oppositely charged tracks consistent with coming from a common vertex and having an invariant mass within $\pm 9 \mathrm{MeV} / c^{2}$ of the nominal $K_{S}^{0}$ mass. For most of the channels involving a $K_{S}^{0}$, we require that the $K_{S}^{0}$ vertex be displaced from the interaction point for the event by at least $0.2 \mathrm{~cm}$ in the plane transverse to the beam axis direction. The $\pi^{0}$ candidates are reconstructed from pairs of photons, each with energy greater than $30 \mathrm{MeV}$, which are required to have an invariant mass $115<m_{\gamma \gamma}<150 \mathrm{MeV} / c^{2}$. The $\pi^{0}$ from a $D^{* 0}$ decay must have momentum between $70 \mathrm{MeV} / c$ and $450 \mathrm{MeV} / c$ in the $\Upsilon(4 S)$ frame, while the $\pi^{0}$ from $D^{0} \rightarrow K^{-} \pi^{+} \pi^{0}$ must have energy greater than $200 \mathrm{MeV}$ in the laboratory frame.

The $D^{*}$ candidates are reconstructed in the decay modes $D^{*+} \rightarrow D^{0} \pi^{+}, D^{*+} \rightarrow D^{+} \pi^{0}, D^{* 0} \rightarrow D^{0} \pi^{0}$, and $D^{* 0} \rightarrow D^{0} \gamma$. A $\pm 3 \sigma$ interval around the nominal mass difference $\Delta m=m\left(D^{*}\right)-m\left(D^{0}\right)$ is used to select $D^{*}$ mesons, where $\sigma$ is the measured mass difference resolution and is equal to $1 \mathrm{MeV} / c^{2}$ for $D^{*+} \rightarrow D^{0} \pi^{+}$and $D^{*+} \rightarrow D^{+} \pi^{0}$ decays, $1.3 \mathrm{MeV} / c^{2}$ for $D^{* 0} \rightarrow D^{0} \pi^{0}$ decays, and $3.3 \mathrm{MeV} / c^{2}$ for $D^{* 0} \rightarrow D^{0} \gamma$ decays. The mode $D^{*+} \rightarrow D^{+} \pi^{0}$ is used only in the reconstruction of decays $B^{0} \rightarrow D^{*-} D^{*+} K_{S}^{0}$ and $B^{+} \rightarrow D^{*-} D^{*+} K^{+}$.

The $D^{0}$ and $D^{+}$mesons are reconstructed in the decay modes $D^{0} \rightarrow K^{-} \pi^{+}, K^{-} \pi^{+} \pi^{0}, K^{-} \pi^{+} \pi^{-} \pi^{+}$, and $D^{+} \rightarrow K^{-} \pi^{+} \pi^{+}$, by selecting track combinations with invariant mass within $\pm 2 \sigma$ of the average measured $D$ mass. The average $D$ mass and the $D$ mass resolution $\sigma$ used in this selection are fitted from the data itself, using a large inclusive sample of $D$ decays. The resolution is equal to $7 \mathrm{MeV} / c^{2}$ for $D^{0} \rightarrow K^{-} \pi^{+}$decays, $13 \mathrm{MeV} / c^{2}$ for $D^{0} \rightarrow K^{-} \pi^{+} \pi^{0}$ decays, $5.7 \mathrm{MeV} / c^{2}$ for $D^{0} \rightarrow K^{-}$ $\pi^{+} \pi^{-} \pi^{+}$decays, and $5.5 \mathrm{MeV} / c^{2}$ for $D^{+} \rightarrow K^{-} \pi^{+} \pi^{+}$ decays. For modes involving two $D^{0}$ mesons, at least one of them is required to decay to $K^{-} \pi^{+}$, except for the decay modes $D^{*-} D^{*+} K_{S}^{0}, D^{*-} D^{*+} K^{+}$, and $D^{*-} D^{0} K^{+}$, which have lower background and for which all combinations are accepted. All $K$ and $\pi$ tracks are required to be well reconstructed in the tracking detectors and to originate from a common vertex. Charged kaon identification, based on the measured Cherenkov angle in the DIRC and the $\mathrm{d} E / \mathrm{d} x$ measurements in the drift chamber and the vertex tracker, is used for most $D$ decay modes, as well as for the $K^{+}$from the $B$ meson decay.

$B$ candidates are reconstructed by combining one $\bar{D}^{(*)}$, one $D^{(*)}$ and one $K$ candidate. A mass-constrained kinematic fit is applied to all intermediate particles $\left(D^{* 0}\right.$, $\left.D^{*+}, D^{0}, D^{+}, K_{S}^{0}, \pi^{0}\right)$. Since the $B$ mesons are produced via $e^{+} e^{-} \rightarrow \Upsilon(4 S) \rightarrow B \bar{B}$, the energy of the $B$ meson in the $\Upsilon(4 S)$ rest frame is given by the beam energy in the center-of-mass frame, $\sqrt{s} / 2$, which is known much more precisely than the energy of the $B$ candidate. Therefore, to isolate the $B$ meson signal, we use two kinematic variables: the difference between the reconstructed energy of the $B$ candidate and the beam energy in the center of mass frame $(\Delta E)$, and the beam energy substituted mass $\left(m_{\mathrm{ES}}\right)$, defined as

$$
m_{\mathrm{ES}}=\sqrt{\left(\frac{\sqrt{s}}{2}\right)^{2}-p_{B}^{* 2}},
$$

where $p_{B}^{*}$ is the momentum of the reconstructed $B$ in the $\Upsilon(4 S)$ frame. Signal events have $m_{\mathrm{ES}}$ close to the nominal $B$ meson mass, $5.279 \mathrm{GeV} / c^{2}$, and $\Delta E$ close to $0 \mathrm{MeV}$. Due to imperfect modeling of the charged $K$ energy loss in the detector material, the central value of $\Delta E$ is slightly shifted away from $0 \mathrm{MeV}$ by an amount $\Delta E_{\text {shift }}=(-5 \pm 1) \mathrm{MeV}$, which is fitted from the data themselves (Figs. 2 (a), 2 (b)). When several candidates are selected in the same event, only the candidate with the lowest $\left|\Delta E-\Delta E_{\text {shift }}\right|$ value is considered ("best candidate"). From Monte Carlo studies, this algorithm is found to give the best reconstruction efficiency and the lowest cross-feed rate between the different $\bar{D}^{(*)} D^{(*)} K$ 
modes; it is found to introduce no bias on the signal extraction, since the latter is performed from the $m_{\mathrm{ES}}$ spectra only. However, in Fig. 2, to avoid the bias on $\Delta E$ inherent to this method, $\Delta E$ spectra are shown without applying this selection.

\section{EVIDENCE FOR $B \rightarrow \bar{D}^{(*)} D^{(*)} K$}

The $m_{\mathrm{ES}}$ and $\Delta E$ spectra of the selected events are shown in Fig. 2 for the sum of all the decay modes, separately for $B^{0}$ and $B^{+}$. The $\Delta E$ spectra are shown for events in the signal region defined by $5.27<m_{\mathrm{ES}}<$ $5.29 \mathrm{GeV} / c^{2}$. Signal events appear in the peak near $0 \mathrm{MeV}$ when reconstructed correctly, while the peak around $-160 \mathrm{MeV}$ is due to $\bar{D}^{*} D K$ and $\bar{D} D^{*} K$ decays reconstructed as $\bar{D} D K$ and to $\bar{D}^{*} D^{*} K$ decays reconstructed as $\bar{D}^{*} D K$ or $\bar{D} D^{*} K$. The $m_{\mathrm{ES}}$ spectra for the signal region are shown for events with $\Delta E$ within $\pm 2.5 \sigma_{\Delta E}$ of the central $\Delta E$ value for the signal. The resolution $\sigma_{\Delta E}$ is determined from the data and is equal to $9.9 \mathrm{MeV}$ for events involving no $D^{* 0}$ and $11.3 \mathrm{MeV}$ for events involving one $D^{* 0}$. For events with two $D^{* 0}$ candidates, the resolution is estimated from the Monte Carlo simulation to be $13.8 \mathrm{MeV}$. As explained above, only the candidate with the lowest $\left|\Delta E-\Delta E_{\text {shift }}\right|$ appears in the $m_{\mathrm{ES}}$ spectra in case of multiple candidates. Both the $m_{\mathrm{ES}}$ spectra for the $\Delta E$ signal region and the $\Delta E$ spectra show clear evidence of a signal. On the contrary, the $m_{\mathrm{ES}}$ spectra for the background control region $\Delta E>50 \mathrm{MeV}$ do not contain any excess of events in the $B$ signal region as expected. When fitting the $m_{\mathrm{ES}}$ spectra, the combinatorial background component is empirically described by a threshold function [11] (henceforth referred to as the ARGUS distribution),

$$
\begin{aligned}
\frac{\mathrm{d} N}{\mathrm{~d} m_{\mathrm{ES}}}= & f\left(m_{\mathrm{ES}} ; A, \zeta\right)=A m_{\mathrm{ES}} \sqrt{1-\frac{m_{\mathrm{ES}}^{2}}{m_{0}^{2}}} \\
& \times \exp \left[-\zeta\left(1-\frac{m_{\mathrm{ES}}^{2}}{m_{0}^{2}}\right)\right],
\end{aligned}
$$

where $m_{0}$ represents the kinematic upper limit and is held fixed at the center-of-mass beam energy $E_{\text {beam }}^{*}=$ $5.291 \mathrm{GeV}$, and $A$ is a normalization factor. The function depends on a free parameter $\zeta$ that is determined from a fit to the $m_{\mathrm{ES}}$ spectrum of the background control region. The number of combinatorial background events in the signal region is then estimated by normalizing the ARGUS distribution to the region $5.22<$ $m_{\mathrm{ES}}<5.27 \mathrm{GeV} / c^{2}$ in the $\Delta E$ slice containing the signal (Figs. 2(c), 2(d)) and extrapolating it to the signal region $5.27<m_{\mathrm{ES}}<5.29 \mathrm{GeV} / c^{2}$. The fitted ARGUS distributions are overlaid on the $m_{\mathrm{ES}}$ spectra of Fig. 2.

The number of background events predicted in the signal region by the fit is $1889 \pm 24$ for neutral $B$ mesons and $2512 \pm 27$ for charged $B$ mesons, while 2712 and 3482 events are observed, giving an excess of $823 \pm 57 B^{0}$ and $970 \pm 65 \mathrm{~B}^{+}$events in the signal region.

\section{DETERMINATION OF BRANCHING FRACTIONS}

In the following, the subscript $k$ will be used to identify the different $B \rightarrow \bar{D}^{(*)} D^{(*)} K$ decay modes (i.e., $\left.\bar{D}^{0} D^{0} K^{+}, D^{*-} D^{0} K^{+}, \ldots\right)$. The subscript $i$ will be used to identify the different decay submodes of the $\bar{D} D$ pair (i.e., $i=K \pi \times K \pi, K \pi \times K \pi \pi^{0}, K \pi \times K 3 \pi, \ldots$ ). The subscript $i k$ will therefore refer to $B$ mode $k$ decaying into $\bar{D} D$ submode $i$.

The $m_{\mathrm{ES}}$ spectra obtained after a $\pm 2.5 \sigma_{\Delta E}$ selection on $\left(\Delta E-\Delta E_{\text {shift }}\right)$ for all the different $\bar{D}^{(*)} D^{(*)} K$ modes are shown in Fig. 3 ( $B^{0}$ decay modes) and Fig. $4\left(B^{+}\right.$decay modes). The corresponding event yields, computed as explained below, are given in Table I. In Figs. 3 and 4 and in Table I, for a given $B$ decay mode the signals from the different $\bar{D} D$ decay submodes have been summed. However, to take advantage of the different signal-to-background ratios of the various submodes, the information from each submode is entered separately in a likelihood function used to calculate the $B \rightarrow \bar{D}^{(*)} D^{(*)} K$ branching fractions. As a first step, the ARGUS distribution shape parameter of each submode, $\zeta_{i k}$, is determined from a maximum likelihood fit to the $m_{\mathrm{ES}}$ spectra of the background control region $\Delta E>50 \mathrm{MeV}$. An ARGUS distribution with the shape parameter $\zeta$ fixed to this value $\zeta_{i k}$ is then fitted to the $m_{\mathrm{ES}}$ distribution for the signal region $\left|\Delta E-\Delta E_{\text {shift }}\right|<2.5 \sigma_{\Delta E}$, excluding from the fit events with $5.27<m_{\mathrm{ES}}<5.29 \mathrm{GeV} / c^{2}$. The factor $A_{i k}$ is calculated so that the function is normalized to the total number of background events and the number of background events, $\mu_{i k}^{\mathrm{bkg}}$, in the signal region for this submode is calculated as

$$
\mu_{i k}^{\mathrm{bkg}}=\int_{5.27}^{5.29} f\left(x ; A_{i k}, \zeta_{i k}\right) \mathrm{d} x .
$$

If $n_{k}$ submodes are used for a given mode, the branching fraction for that mode is then extracted by maximizing the following likelihood:

$$
L_{k}=\prod_{i=1}^{n_{k}} \frac{\mu_{i k}{ }^{N_{i k}} e^{-\mu_{i k}}}{N_{i k} !}
$$

where $N_{i k}$ and $\mu_{i k}$ are the observed and predicted number of events, respectively, in the signal region. $\mu_{i k}$ is the sum of three contributions:

- the predicted signal $\mu_{i k}^{S}$, which is the product of the (unknown) branching fraction $\mathcal{B}_{k}$ of decay mode $k$, the reconstruction efficiency $\epsilon_{i k}$, the intermediate branching fractions $\mathcal{B}_{i}^{\bar{D} D}$, and the number of $B \bar{B}$ events, $N_{B \bar{B}}$, assuming that the number of $B^{0} \bar{B}^{0}$ meson pairs produced at the $\Upsilon(4 S)$ resonance is equal to the number of $B^{+} B^{-}$pairs:

$$
\mu_{i k}^{S}=\mathcal{B}_{k} \times N_{B \bar{B}} \times \epsilon_{i k} \times \mathcal{B}_{i}^{\bar{D} D}
$$

- the number of combinatorial background events, $\mu_{i k}^{\mathrm{bkg}}$, determined as described above (Eq. 5); 

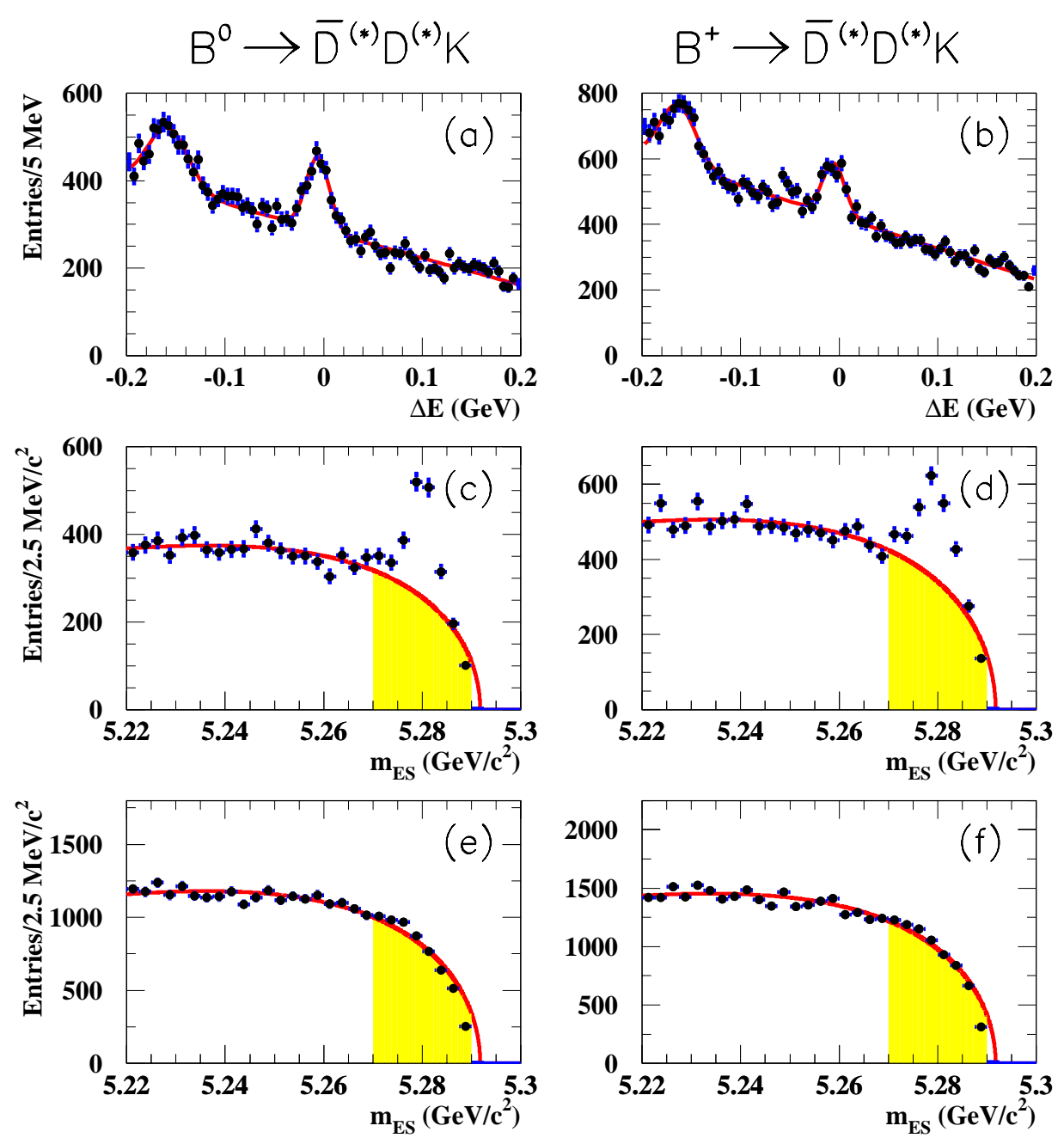

FIG. 2: The $\Delta E$ and $m_{\mathrm{ES}}$ spectra (a,c,e) for the sum of all the $B^{0} \rightarrow \bar{D}^{(*)} D^{(*)} K$ modes and (b,d,f) for the sum of all the $B^{+} \rightarrow \bar{D}^{(*)} D^{(*)} K$ modes. (a,b): $\Delta E$ for $5.27<m_{\mathrm{ES}}<5.29 \mathrm{GeV} / c^{2}$. (c,d): $m_{\mathrm{ES}}$ for $\left|\Delta E-\Delta E_{\text {shift }}\right|<2.5 \sigma_{\Delta E}$. (e,f): $m_{\mathrm{ES}}$ for $\Delta E>50 \mathrm{MeV}$ (background control region). The curves superimposed on the $m_{\mathrm{ES}}$ spectra correspond to the background fits described in the text and the shaded regions represent the background in the signal region $5.27<m_{\mathrm{ES}}<5.29 \mathrm{GeV} / c^{2}$.

- the peaking background $\mu_{i k}^{\text {peak }}$ from other $B \rightarrow$ $\bar{D}^{(*)} D^{(*)} K$ decay modes. The cross-feed between different $\bar{D} D$ decay submodes is found to be negligible and $\mu_{i k}^{\text {peak }}$ is therefore calculated as

$$
\mu_{i k}^{\text {peak }}=\sum_{l \neq k} \mathcal{B}_{l} \times N_{B \bar{B}} \times \epsilon^{\prime}(i l \rightarrow i k) \times \mathcal{B}_{i}^{\bar{D} D}
$$

where $\epsilon^{\prime}(i l \rightarrow i k)$ is the cross-feed matrix element that represents the probability for $B$ mode $l$ to be reconstructed as $B$ mode $k$ for $\bar{D} D$ decay submode $i$. The only significant cross-feed is observed between decay modes where a fake $D^{* 0}$ re- places a real $D^{*+}$ or a real $D^{0}$, for instance between $D^{*-} D^{0} K^{+}$and $\bar{D}^{* 0} D^{0} K^{+}$, or between $\bar{D}^{* 0} D^{0} K^{+}$ and $\bar{D}^{0} D^{* 0} K^{+}$.

The branching fractions $\mathcal{B}_{k}$ for the sets of decay modes that have significant cross-feed are simultaneously fit, by maximizing the product $\prod_{k} L_{k}$ of the corresponding likelihood functions.

The $D^{*}$ and $D$ branching fractions used in the branching fraction calculation are summarized in Table II [12]. Branching fractions for decay modes reconstructed with a $K_{S}^{0}$ are calculated for neutral $K$ mesons, including $K_{L}^{0}$. The selection efficiencies and the cross-feed matrices for each mode are obtained from a detailed Monte Carlo sim- 

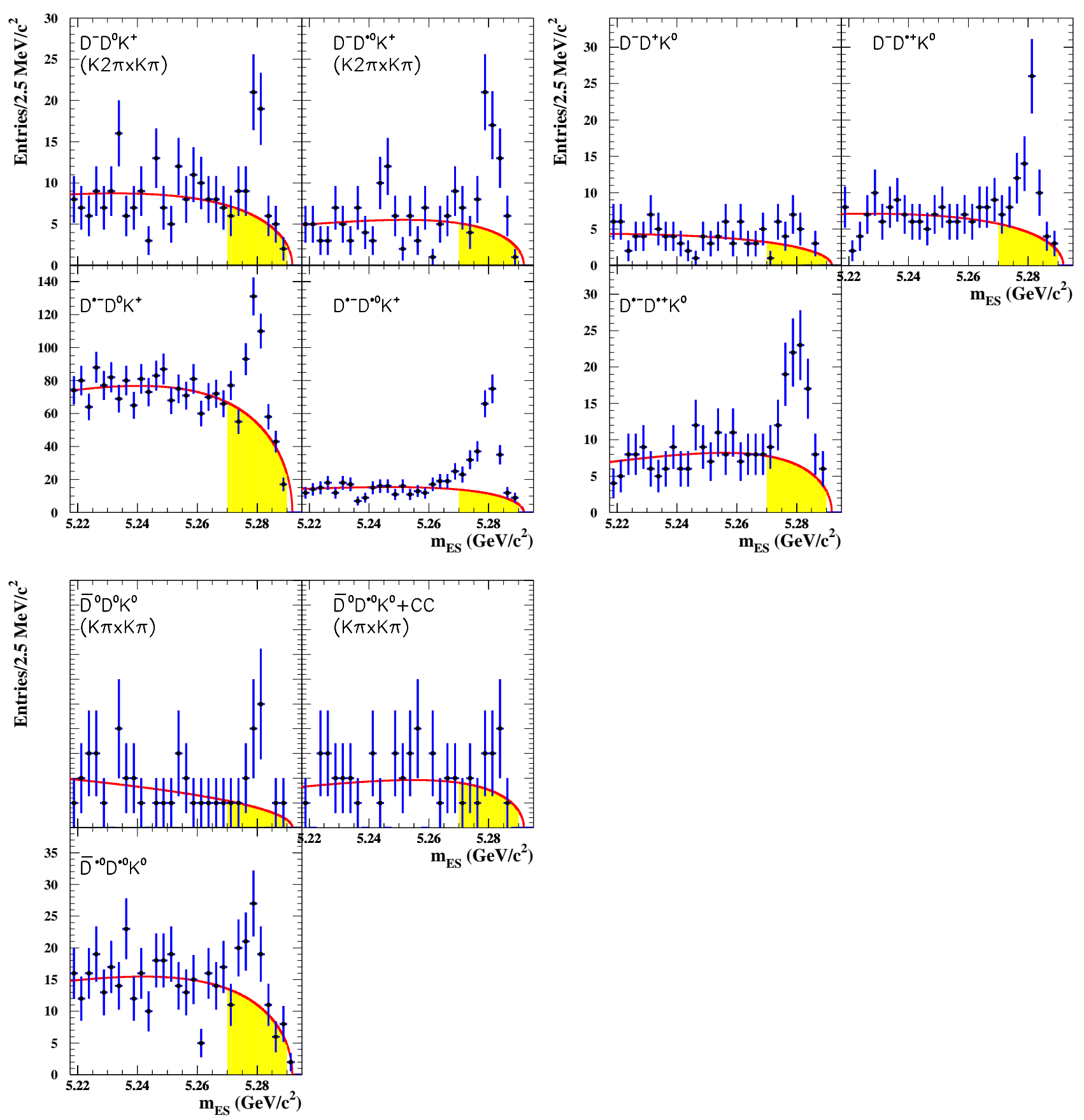

FIG. 3: The $m_{\mathrm{ES}}$ spectra of the ten $B^{0} \rightarrow \bar{D}^{(*)} D^{(*)} K$ modes. For each mode, all the $D$ decay submodes used in the analysis have been summed, except for $B$ modes for which the $\bar{D} \times D$ decay mode is listed explicitly on the plot. The curves correspond to the background fits described in the text and the shaded regions represent the background in the signal region. Upper left: pure external $W$-emission (spectator) decays $B^{0} \rightarrow D^{(*)-} D^{(*) 0} K^{+}$. Upper right: external+internal $W$-emission decays $B^{0} \rightarrow D^{(*)-} D^{(*)+} K_{S}^{0}$. Lower left: pure internal $W$-emission (color-suppressed) decays $B^{0} \rightarrow \bar{D}^{(*) 0} D^{(*) 0} K_{S}^{0}$.

ulation, in which the detector response is modeled with the Geant4 program [13]. The simulated event samples of $B \rightarrow \bar{D}^{(*)} D^{(*)} K$ decays used for the efficiency calculation are generated according to a phase space model.
For each decay submode, samples of about 15000 signal events have been produced. In addition, data are used whenever possible to determine detector performance: tracking efficiencies are determined by identifying tracks 

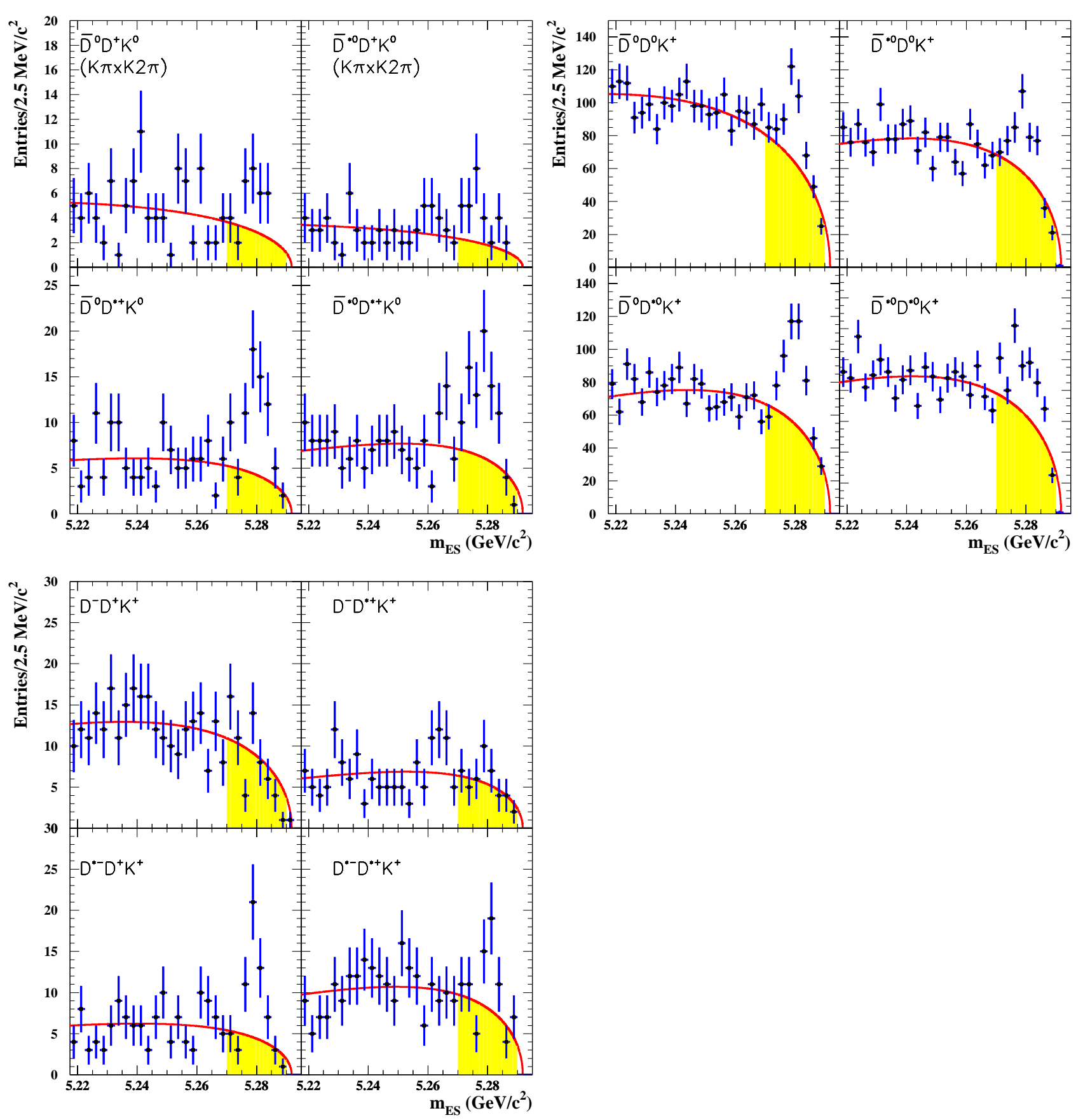

FIG. 4: The $m_{\mathrm{ES}}$ spectra of the twelve $B^{+} \rightarrow \bar{D}^{(*)} D^{*} K$ modes. For each mode, all the $D$ decay submodes used in the analysis have been summed, except for $B$ modes for which the $\bar{D} \times D$ decay mode is listed explicitly on the plot. The curves correspond to the background fits described in the text and the shaded regions represent the background in the signal region. Upper left: pure external $W$-emission (spectator) decays $B^{+} \rightarrow \bar{D}^{(*) 0} D^{(*)+} K_{S}^{0}$. Upper right: external+internal $W$-emission decays $B^{+} \rightarrow \bar{D}^{(*) 0} D^{(*) 0} K^{+}$. Lower left: pure internal $W$-emission (color-suppressed) decays $B^{+} \rightarrow D^{(*)-} D^{(*)+} K^{+}$.

in the silicon vertex detector and measuring the fraction that is well reconstructed in the drift chamber; the kaon identification efficiency is estimated from a sample of $D^{*+} \rightarrow D^{0} \pi^{+}, D^{0} \rightarrow K^{-} \pi^{+}$decays; the $\gamma$ and $\pi^{0}$ ef- ficiencies are measured by comparing the ratio of events, $N\left(\tau^{+} \rightarrow \bar{\nu}_{\tau} h^{+} \pi^{0}\right) / N\left(\tau^{+} \rightarrow \bar{\nu}_{\tau} h^{+} \pi^{0} \pi^{0}\right)$, to the published branching fractions [14]. Typical efficiencies range from $20 \%$ for $B^{+} \rightarrow \bar{D}^{0} D^{0} K^{+}$with both $D^{0}$ mesons decay- 
TABLE I: Number of events and branching fractions for each mode. The excess (column 4) is the difference between the total yield observed in the signal region $5.27<m_{\mathrm{ES}}<5.29 \mathrm{GeV} / c^{2}$ and the combinatorial background. It includes the contribution from the signal itself and from the cross-feed from the other $\bar{D}^{(*)} D^{(*)} K$ modes. The number of cross-feed events, computed from the cross-feed matrix and from the measured $B \rightarrow \bar{D}^{(*)} D^{(*)} K$ branching fractions, is given in column 5 . When omitted, the predicted number of cross-feed events is smaller than 5 and has been neglected in the branching fraction calculations, as explained in the text. The first error on each branching fraction is the statistical uncertainty and the second one is the systematic uncertainty. For the decay modes with a significance $S / \sqrt{B}$ smaller than 4 , a $90 \%$ confidence level (C.L.) upper limit is also derived. Here, $B$ is the sum of the combinatorial background and of cross-feed, while $S=N-B$, where $N$ is the total yield in the signal region. The decay mode $B^{+} \rightarrow \bar{D}^{* 0} D^{0} K^{+}$has a large number of signal events but its significance is lower than 4 because of the large cross-feed from $B^{+} \rightarrow \bar{D}^{0} D^{* 0} K^{+}$and $B^{0} \rightarrow D^{*-} D^{0} K^{+}$. The fractional statistical error on the branching fractions cannot be directly related to the fractional statistical error on the excess since the different decay submodes of the $\bar{D} D$ pair (not detailed in the table) enter with different statistical weights in the branching fraction calculation, while the yields given here are a raw sum over all the $\bar{D} D$ decay submodes. The statistical uncertainty on the background, dominated by the uncertainty on the ARGUS shape parameter $\zeta$, is incorporated in the systematic error on the branching fractions.

\begin{tabular}{|c|c|c|c|c|c|c|}
\hline $\begin{array}{l}B \text { decay } \\
\text { mode }\end{array}$ & \begin{tabular}{|c|} 
Total yield \\
$N$ in the \\
signal region \\
\end{tabular} & \begin{tabular}{|c|} 
Estimated \\
combinatorial \\
background
\end{tabular} & \begin{tabular}{|c|} 
Excess $=$ \\
Signal + \\
cross-feed \\
\end{tabular} & $\begin{array}{c}\text { cross- } \\
\text { feed }\end{array}$ & $\begin{array}{c}\text { Branching } \\
\text { fraction } \\
(\%) \\
\end{array}$ & $\begin{array}{l}90 \% \text { C.L. } \\
\text { upper } \\
\text { limit ( } \%) \\
\end{array}$ \\
\hline \multicolumn{7}{|c|}{$B^{0}$ decays through external $W$-emission amplitudes } \\
\hline$B^{0} \rightarrow D^{-} D^{0} K^{+}$ & \begin{tabular}{|l|l|}
599 &
\end{tabular} & $479 \pm 12$ & $120 \pm 27$ & - & $0.17 \pm 0.03 \pm 0.03$ & \\
\hline$B^{0} \rightarrow D^{-} D^{* 0} K^{+}$ & 468 & $337 \pm 10$ & $131 \pm 24$ & - & $0.46 \pm 0.07 \pm 0.07$ & \\
\hline$B^{0} \rightarrow D^{*-} D^{0} K^{+}$ & 584 & $399 \pm 11$ & $185 \pm 27$ & - & 40.04 & \\
\hline$B^{0} \rightarrow D^{*-} D^{* 0} K^{+}$ & 289 & $84 \pm 5$ & $205 \pm 18$ & - & $1.18 \pm 0.10 \pm 0.17$ & \\
\hline \multicolumn{7}{|c|}{$B^{0}$ decays through external+internal $W$-emission amplitudes } \\
\hline$B^{0} \rightarrow D^{-} D^{+} K^{0}$ & \begin{tabular}{|l|l}
26 &
\end{tabular} & $19 \pm 2$ & $7 \pm 5$ & - & $0.08_{-0.05}^{+0.06} \pm 0.03$ & 0.17 \\
\hline$B^{0} \rightarrow D^{*-} D^{+} K^{0}+D^{-} D^{*+} K^{0}$ & 84 & $34 \pm 3$ & $50 \pm 10$ & - & $0.65 \pm 0.12 \pm 0.10$ & \\
\hline$B^{0} \rightarrow D^{*-} D^{*+} K^{0}$ & 116 & $48 \pm 4$ & $68 \pm 11$ & - & $0.88_{-0.14}^{+0.15} \pm 0.13$ & \\
\hline \multicolumn{7}{|c|}{$B^{0}$ decays through internal $W$-emission amplitudes } \\
\hline$B^{0} \rightarrow \bar{D}^{0} D^{0} K^{0}$ & \begin{tabular}{|l|l}
175 &
\end{tabular} & $173 \pm 7$ & $2 \pm 15$ & - & $0.08 \pm 0.04 \pm 0.02$ & 0.14 \\
\hline$B^{0} \rightarrow \bar{D}^{0} D^{* 0} K^{0}+\bar{D}^{* 0} D^{0} K^{0}$ & 248 & $225 \pm 8$ & $23 \pm 18$ & - & $0.17_{-0.13}^{+0.14} \pm 0.07$ & 0.37 \\
\hline$B^{0} \rightarrow \bar{D}^{* 0} D^{* 0} K^{0}$ & 123 & $81 \pm 6$ & $42 \pm 13$ & 19.8 & $0.33_{-0.20}^{+0.21} \pm 0.14$ & 0.66 \\
\hline \multicolumn{7}{|c|}{$B^{+}$decays through external $W$-emission amplitudes } \\
\hline$B^{+} \rightarrow \bar{D}^{0} D^{+} K^{0}$ & \begin{tabular}{|l|l}
367 &
\end{tabular} & $317 \pm 9$ & $50 \pm 21$ & - & $0.18 \pm 0.07 \pm 0.04$ & 0.28 \\
\hline$B^{+} \rightarrow \bar{D}^{* 0} D^{+} K^{0}$ & 216 & $175 \pm 7$ & $41 \pm 16$ & 9.6 & $0.41_{-0.14}^{+0.15} \pm 0.08$ & 0.61 \\
\hline$B^{+} \rightarrow \bar{D}^{0} D^{*+} K^{0}$ & 77 & $31 \pm 3$ & $46 \pm 9$ & - & $0.52_{-0.09}^{+0.10} \pm 0.07$ & \\
\hline$B^{+} \rightarrow \bar{D}^{* 0} D^{*+} K^{0}$ & 89 & $43 \pm 4$ & $46 \pm 10$ & 9.0 & $0.78_{-0.21}^{+0.23} \pm 0.14$ & \\
\hline \multicolumn{7}{|c|}{$B^{+}$decays through external+internal $W$-emission amplitudes } \\
\hline$B^{+} \rightarrow \bar{D}^{0} D^{0} K^{+}$ & \begin{tabular}{|l|l|}
627 &
\end{tabular} & $469 \pm 11$ & $158 \pm 27$ & - & $0.19 \pm 0.03 \pm 0.03$ & \\
\hline$B^{+} \rightarrow \bar{D}^{* 0} D^{0} K^{+}$ & 552 & $411 \pm 11$ & $141 \pm 26$ & 75.3 & $0.18_{-0.06}^{+0.07} \pm 0.04$ & 0.38 \\
\hline$B^{+} \rightarrow \bar{D}^{0} D^{* 0} K^{+}$ & 623 & $402 \pm 11$ & $221 \pm 27$ & 37.1 & $0.47 \pm 0.07 \pm 0.07$ & \\
\hline$B^{+} \rightarrow \bar{D}^{* 0} D^{* 0} K^{+}$ & 675 & $468 \pm 15$ & $207 \pm 30$ & 66.6 & ${ }_{0}^{1} \pm 0.12$ & \\
\hline \multicolumn{7}{|c|}{$B^{+}$decays through internal $W$-emission amplitudes } \\
\hline$B^{+} \rightarrow D^{-} D^{+} K^{+}$ & \begin{tabular}{|l|l}
64 &
\end{tabular} & $65 \pm 4$ & $-1 \pm 9$ & - & $0.00 \pm 0.03 \pm 0.01$ & 0.04 \\
\hline$B^{+} \rightarrow D^{-} D^{*+} K^{+}$ & 45 & $39 \pm 4$ & $6 \pm 8$ & - & $0.02 \pm 0.02 \pm 0.01$ & 0.07 \\
\hline$B^{+} \rightarrow D^{*-} D^{+} K^{+}$ & 64 & $32 \pm 3$ & $32 \pm 9$ & - & $0.15 \pm 0.03 \pm 0.02$ & \\
\hline$B^{+} \rightarrow D^{*-} D^{*+} K^{+}$ & 83 & $60 \pm 4$ & $23 \pm 10$ & - & $0.09 \pm 0.04 \pm 0.02$ & 0.18 \\
\hline
\end{tabular}

ing to $K^{-} \pi^{+}$, to less than $1 \%$ for $B^{+} \rightarrow D^{*-} D^{*+} K^{+}$ $\left(D^{*+} \rightarrow D^{0} \pi^{+}, D^{*-} \rightarrow \bar{D}^{0} \pi^{-}\right)$with $D^{0}$ mesons decaying to $K^{-} \pi^{+} \pi^{0}$ or $K^{-} \pi^{+} \pi^{-} \pi^{+}$.

\section{SYSTEMATIC STUDIES}

Due to the large number of $K^{ \pm}$mesons and to the large track multiplicities involved in the decays $B \rightarrow$ $\bar{D}^{(*)} D^{(*)} K$, the dominant systematic uncertainties come from our level of understanding of the charged kaon iden- tification and of the charged-particle tracking efficiencies. Both systematic uncertainties are estimated for each track and are given in Table III. Another important systematic is the uncertainty linked to the background description. One of its components is from the uncertainty on the number of background events and is dominated by the uncertainty on the ARGUS shape parameter $\zeta$. The relative error on the branching fractions associated with this component varies from $5 \%$ up to $20 \%$ depending on the mode and is uncorrelated from one mode to another. The other component is from the 
TABLE II: Submode branching fractions used in the analysis [12]. The errors on $\mathcal{B}\left(D^{0} \rightarrow K^{-} \pi^{+} \pi^{0}\right)$ and $\mathcal{B}\left(D^{0} \rightarrow\right.$ $\left.K^{-} \pi^{+} \pi^{-} \pi^{+}\right)$correlated to the error on $\mathcal{B}\left(D^{0} \rightarrow K^{-} \pi^{+}\right)$are indicated separately with the subscript $K \pi$.

\begin{tabular}{|l|c|}
\hline Mode & $\mathcal{B}(\%)$ \\
\hline \hline$D^{0} \rightarrow K^{-} \pi^{+}$ & $3.80 \pm 0.09$ \\
$D^{0} \rightarrow K^{-} \pi^{+} \pi^{0}$ & $13.10 \pm 0.84 \pm 0.31_{K \pi}$ \\
$D^{0} \rightarrow K^{-} \pi^{+} \pi^{-} \pi^{+}$ & $7.46 \pm 0.30 \pm 0.18_{K \pi}$ \\
\hline$D^{+} \rightarrow K^{-} \pi^{+} \pi^{+}$ & $9.1 \pm 0.6$ \\
\hline$D^{*+} \rightarrow D^{0} \pi^{+}$ & $67.7 \pm 0.5$ \\
$D^{*+} \rightarrow D^{+} \pi^{0}$ & $30.7 \pm 0.5$ \\
\hline$D^{* 0} \rightarrow D^{0} \pi^{0}$ & $61.9 \pm 2.9$ \\
$D^{* 0} \rightarrow D^{0} \gamma$ & $38.1 \pm 2.9$ \\
\hline$K_{S}^{0} \rightarrow \pi^{+} \pi^{-}$ & $68.60 \pm 0.27$ \\
\hline
\end{tabular}

end point $m_{0}$ of the ARGUS distribution. Changing $m_{0}$ by $\pm 1 \mathrm{MeV}$ results in a $\pm 1.4 \%$ change of the fitted combinatorial background. The associated fractional error on each branching fractions is estimated to be $\pm 3.5 \%$ in average and is correlated between all the modes. Other systematic uncertainties are due to uncertainties on the $D$ and $D^{*}$ branching fractions, the $\pi^{0}$ reconstruction efficiencies, the $D$ vertex fit quality requirements, and the $\Delta E$ resolution used to define the signal box, as well as the statistical uncertainty on the efficiency due to the finite size of the Monte Carlo simulation samples and the uncertainty on the number of $B \bar{B}$ events in the data sample. The different contributions to the systematic uncertainties on the branching fractions are summarized in Table III.

Possible decay model dependences of the efficiencies were also studied by generating the decays $B^{0} \rightarrow$ $D^{*-} D_{s 1}^{+}$and $B^{0} \rightarrow D^{*-} D_{s 1}^{\prime+}\left(D_{s 1}^{+}, D_{s 1}^{\prime+} \rightarrow D^{* 0} K^{+}\right)$, where $D_{s 1}^{+}$is the narrow $(\Gamma \stackrel{\mathrm{MeV}, m=}{=}$, $\left.2535.35 \mathrm{MeV} / c^{2}\right)$ orbitally excited $1^{+}$state of the $D_{s J}$ system and $D_{s 1}^{\prime+}$ is a wide $\left(\Gamma=250 \mathrm{MeV}, m=2560 \mathrm{MeV} / c^{2}\right)$ $D_{s J}$ resonance. The efficiency for reconstructing these modes was compared to the efficiency found for $B^{0} \rightarrow$ $D^{*-} D^{* 0} K^{+}$decays generated with a phase-space model. We found no statistically significant difference in efficiencies; we assign a systematic uncertainty equal to the statistical error of the ratio $(5 \%)$.

\section{SEARCH FOR RESONANT SUBSTRUCTURE}

$B \rightarrow \bar{D}^{(*)} D^{(*) 0} K^{+}$decay modes are used to probe the possible presence of intermediate $D_{s J}$ resonances decaying into $D^{(*) 0} K^{+}$, where $D_{s J}$ are P-wave excitations of the $c \bar{s}$ system. In the heavy-quark (charm) mass limit, the spin of the heavy quark decouples, and both the spin $J$ of the meson and the total angular momentum (spin plus orbital) $j_{q}$ of the light quark become good quantum numbers $[15,16]$. There are four $\mathrm{P}$-wave states with the following spin-parity and light-quark angular momenta: $0^{+}\left(j_{q}=1 / 2\right), 1^{+}\left(j_{q}=1 / 2\right), 1^{+}\left(j_{q}=3 / 2\right)$, $2^{+}\left(j_{q}=3 / 2\right)$. The two $j_{q}=3 / 2$ states can only undergo D-wave decay, and therefore have narrow widths. The remaining $j_{q}=1 / 2$ states decay via S-waves and are expected to be quite broad. Their masses are predicted to be $\approx 2.48 \mathrm{GeV} / c^{2}\left(0^{+}\right)$and $\approx 2.55 \mathrm{GeV} / c^{2}\left(1^{+}\right)$, while their widths are predicted to be a few hundred $\mathrm{MeV}$ [17]. However, the recent observation by the BABAR collaboration of a narrow state decaying to $D_{s}^{+} \pi^{0}$, with a mass of $(2316.8 \pm 0.4) \mathrm{MeV} / c^{2}$ (statistical error only) [19], would contradict these predictions and could indicate that the $J^{P}=0^{+}$state has a mass lower than the $D^{(*)} K$ threshold; if this interpretation is confirmed, the $0^{+}$state would therefore not contribute to the $B \rightarrow \bar{D}^{(*)} D^{(*)} K$ final state.

In the analysis described below, the two narrow resonances, $D_{s 1}^{+}(2536)$ and $D_{s J}^{+}(2573)$, are considered. The full Dalitz plot for the decay $B^{0} \rightarrow D^{*-} D^{* 0} K^{+}$is also examined.

\section{A. $D_{s 1}^{+}(2536)$}

$D_{s 1}^{+}(2536)$ is the most probable resonance to contribute to $B \rightarrow \bar{D}^{(*)} D^{(*)} K$ decays. It has already been observed and its measured parameters are $m=(2535.35 \pm$ $0.60) \mathrm{MeV} / c^{2}, \Gamma<2.3 \mathrm{MeV}, J^{P}=1^{+}$, and $j_{q}=\frac{3}{2}[12]$. Because of conservation of parity and angular momentum, only the decays $D_{s 1}^{+}(2536) \rightarrow D^{*} K$ are allowed. In this analysis, a search is made for the $D_{s 1}^{+}(2536)$ in the final state $D^{* 0} K^{+}$in the four decay modes $B^{0} \rightarrow$ $D^{-} D^{* 0} K^{+}, B^{0} \rightarrow D^{*-} D^{* 0} K^{+}, B^{+} \rightarrow \bar{D}^{0} D^{* 0} K^{+}$, and $B^{+} \rightarrow \bar{D}^{* 0} D^{* 0} K^{+}$. This resonance is not reconstructed in the $D^{*+} K_{S}^{0}$ final state due to its lower reconstruction efficiency.

Figure 5(a) shows the distribution of the variable $\Delta m=m\left(D^{* 0} K^{+}\right)-m\left(D^{* 0}\right)$ for the events reconstructed in the signal region $\left(5.27<m_{\mathrm{ES}}<5.29 \mathrm{GeV} / c^{2}\right)$ for these four decay modes. The distribution is fitted with a Gaussian function describing the signal. The combinatorial background is represented by a threshold function defined as

$$
g(\Delta m)=N\left(\Delta m-\Delta m_{0}\right)^{\beta} e^{\alpha\left(\Delta m-\Delta m_{0}\right)} .
$$

The parameters of the Gaussian function (mean value $\Delta m_{1}=527.9 \mathrm{MeV} / c^{2}$ and standard deviation $\sigma_{\Delta m}=$ $3.5 \mathrm{MeV} / c^{2}$ ) are fixed to the values obtained from a fit to the same distribution resulting from the reconstruction of inclusive $D_{s 1}^{+}(2536) \rightarrow D^{* 0} K^{+}$decays in a large sample of events. This procedure yields an estimated signal of $28_{-7}^{+8} D_{s 1}^{+}(2536) \rightarrow D^{* 0} K^{+}$events out of $764 \pm 50$ $B \rightarrow \bar{D}^{(*)} D^{* 0} K^{+}$events.

In order to extract upper limits on the contribution of $D_{s 1}^{+}(2536)$ to $B \rightarrow \bar{D}^{(*)} D^{(*)} K$ decays, the same method is applied to the four individual decay modes, as shown in Fig. 6(a). The region $519<\Delta m<537 \mathrm{MeV} / c^{2}$, illustrated by the dashed lines in Fig. 5(a), is defined as 
TABLE III: Fractional systematic uncertainties on efficiencies and branching fractions.

\begin{tabular}{|l|l|}
\hline Item & Fractional uncertainty on efficiency or branching fraction \\
\hline \hline Charged track reconstruction & $0.8 \%$ per track for tracks with more that 12 hits required in the Drift Chamber \\
& $1.2 \%$ per track for tracks without Drift Chamber requirement \\
\hline$K_{S}^{0}$ reconstruction & $2.5 \%$ per $K_{S}^{0}$, added in quadrature to the track reconstruction error \\
\hline$\pi^{0}$ reconstruction & $5.1 \%$ per $\pi^{0}$ \\
\hline$\gamma$ from $D^{* 0} \rightarrow D^{0} \gamma$ & $5.1 \%$ per $\gamma$ (correlated with the $\pi^{0}$ systematic) \\
\hline$K^{ \pm}$identification & $2.5 \%$ per $K^{ \pm}$ \\
\hline Vertex & $1.3 \%$ per 2 track vertex \\
reconstruction & $3.1 \%$ per 3 track vertex \\
& $5.7 \%$ per 4 track vertex \\
\hline$\sigma(\Delta E)$ & $2 \%$ for modes with zero or one $D^{* 0}$ \\
& $5 \%$ for modes with two $D^{* 0}$ s \\
\hline Background description & $5 \%$ to $20 \%($ ARG $\mathrm{Sh}$ she parameter $\zeta$, mode dependent) \\
\hline Monte Carlo statistics & $3.5 \%$ (end point $\left.m_{0}\right)$ \\
\hline Intermediate br. fraction & $2 \%$ to $10 \%$ per $\bar{D} D$ submode (mode and submode dependent) \\
\hline Number of $B \bar{B}$ & see Table II \\
\hline Decay model & $1.1 \%$ \\
\hline
\end{tabular}

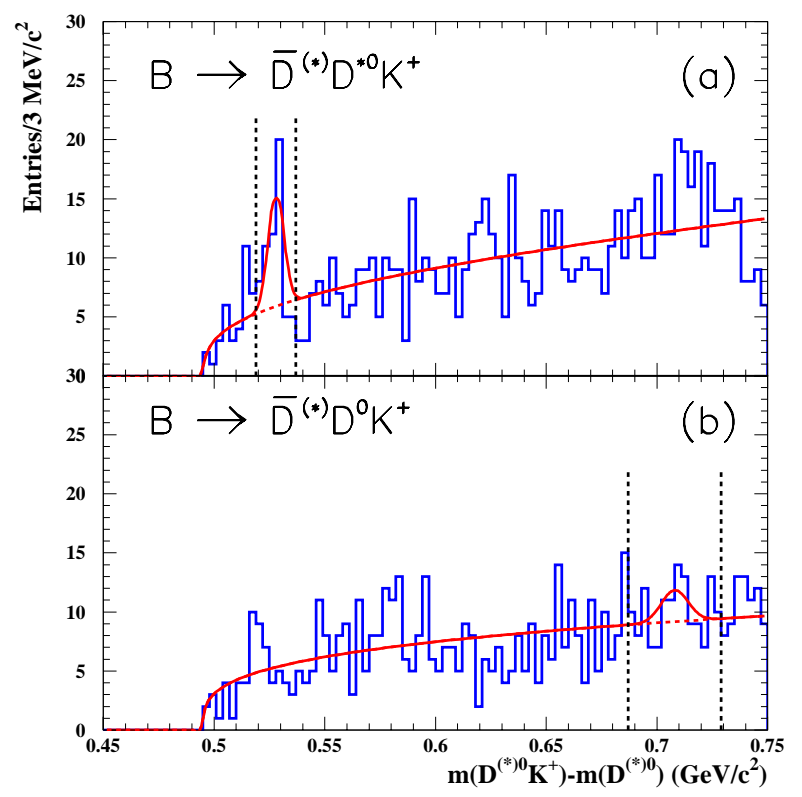

FIG. 5: (a) $\Delta m=m\left(D^{* 0} K^{+}\right)-m\left(D^{* 0}\right)$ distribution for events reconstructed in the $B \rightarrow \bar{D}^{(*)} D^{* 0} K^{+}$signal regions. (b) $\Delta m=m\left(D^{0} K^{+}\right)-m\left(D^{0}\right)$ distribution for events reconstructed in the $B \rightarrow \bar{D}^{(*)} D^{0} K^{+}$signal regions.

the signal region and the number of combinatorial background events in this region is estimated from the fit by integrating the background function $g$ defined in Eq. 9 . The total number of events observed in the signal box is compared to the expected combinatorial background when extracting the limits. Table IV summarizes the re- sults obtained and gives a $90 \%$ confidence level (C.L.) upper limit on the quantity $R_{2536}$,

$$
R_{2536}=\frac{n\left(B \rightarrow \bar{D}^{(*)} D_{s 1}^{+}(2536)\right) \times \mathcal{B}\left(D_{s 1}^{+}(2536) \rightarrow D^{* 0} K^{+}\right)}{n\left(B \rightarrow \bar{D}^{(*)} D^{* 0} K^{+}\right)} .
$$

where $n\left(B \rightarrow \bar{D}^{(*)} D_{s 1}^{+}(2536)\right) \times \mathcal{B}\left(D_{s 1}^{+}(2536) \rightarrow D^{* 0} K^{+}\right)$ and $n\left(B \rightarrow \bar{D}^{(*)} D^{* 0} K^{+}\right)$are the observed number of signal events. Using the $B \rightarrow \bar{D}^{(*)} D^{* 0} K^{+}$ branching fraction measurements of Table I, these results can be compared to the only existing measurement of inclusive $D_{s 1}^{+}(2536)$ production in $B$ decays, $\mathcal{B}\left(B \rightarrow D_{s 1}^{+}(2536) X\right)<0.95 \%$ at $90 \%$ C.L. [18].

TABLE IV: $D_{s 1}^{+}(2536) \rightarrow D^{* 0} K^{+}$contributions to $B \rightarrow$ $\bar{D}^{(*)} D^{* 0} K^{+}$decays.

\begin{tabular}{|l|c|c|c|}
\hline$B$ decay mode & $\begin{array}{c}\text { Total yield } \\
\text { in } D_{s 1}^{+}(2536) \\
\text { signal region }\end{array}$ & $\begin{array}{c}R_{2536} \\
\text { Estimated }\end{array}$ & $\begin{array}{c}\text { background } \\
\text { upper limit }\end{array}$ \\
\hline$D^{-} D^{* 0} K^{+}$ & 16 & $7.8 \pm 0.6$ & $11.6 \%$ \\
$D^{*-} D^{* 0} K^{+}$ & 13 & $7.3 \pm 0.6$ & $5.8 \%$ \\
$\bar{D}^{0} D^{* 0} K^{+}$ & 12 & $11.1 \pm 0.8$ & $3.1 \%$ \\
$\bar{D}^{* 0} D^{* 0} K^{+}$ & 20 & $8.7 \pm 0.5$ & $9.1 \%$ \\
\hline
\end{tabular}

\section{B. $D_{s J}^{+}(2573)$}

The contribution of the $D_{s J}^{+}(2573)$ resonance to $B \rightarrow$ $\bar{D}^{(*)} D^{(*)} K$ decays is also studied. This resonance is thought to be the other narrow state in the $j_{q}=3 / 2$ orbitally excited $D_{s J}$ doublet, together with the $D_{s 1}^{+}(2536)$. The world average values of its mass and width are 

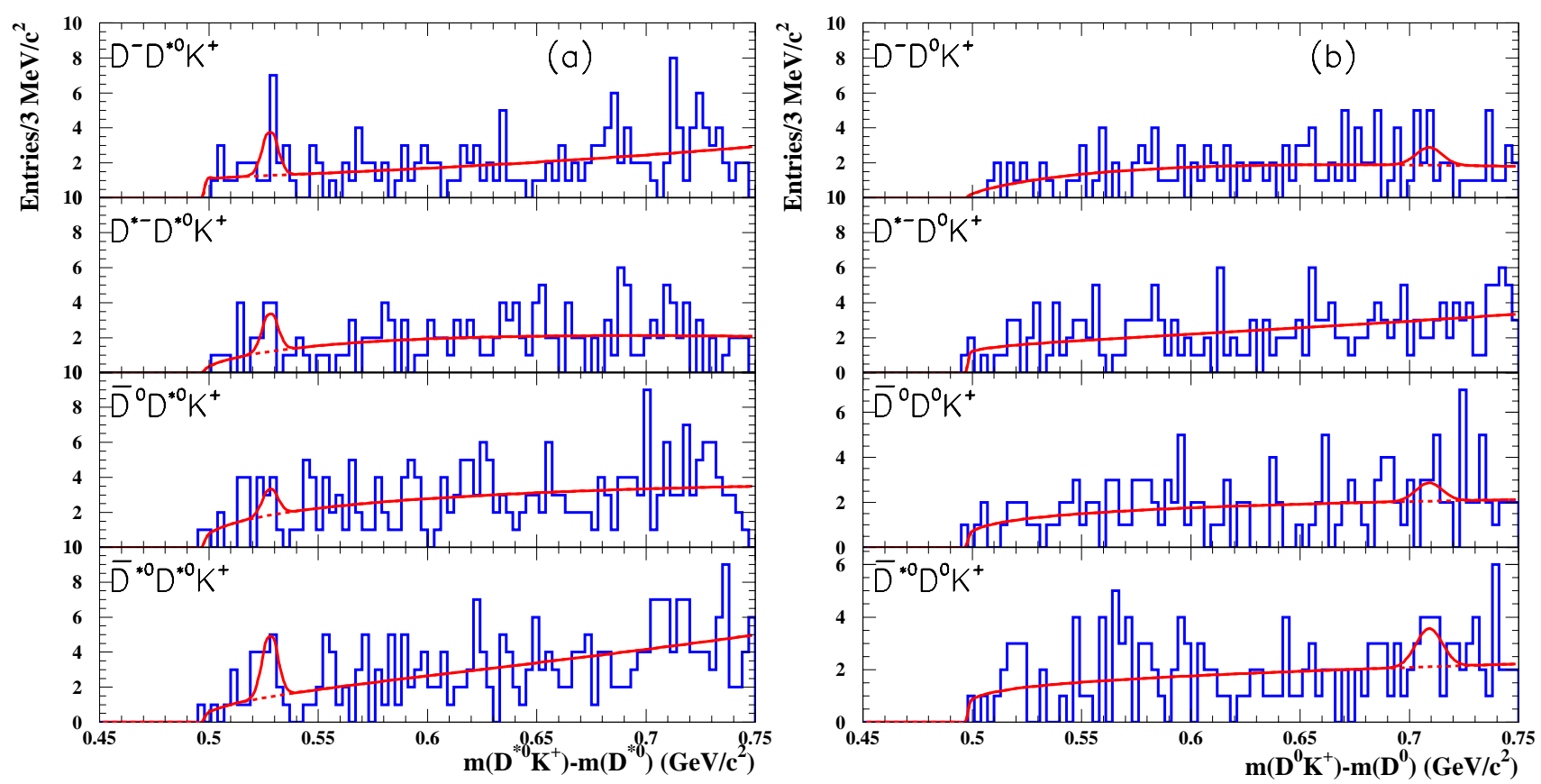

FIG. 6: (a) $\Delta m=m\left(D^{* 0} K^{+}\right)-m\left(D^{* 0}\right)$ distributions of the events reconstructed in the four $B \rightarrow \bar{D}^{(*)} D^{* 0} K^{+}$decay modes, with $5.27<m_{\mathrm{ES}}<5.29 \mathrm{GeV} / c^{2}$. (b) $\Delta m=m\left(D^{0} K^{+}\right)-m\left(D^{0}\right)$ distributions of the events reconstructed in the four $B \rightarrow \bar{D}^{(*)} D^{0} K^{+}$decay modes, with $5.27<m_{\mathrm{ES}}<5.29 \mathrm{GeV} / c^{2}$. These distributions are fit with the sum of a threshold function $f$ (Eq. 9) for the background components and a Gaussian function for the $D_{s 1}(2536)$ and $D_{s J}(2573)$ components. The mean value and standard deviation of the Gaussian distributions have been fixed to the values obtained from a fit to the inclusive $D_{s 1}(2536)$ and $D_{s J}(2573)$ samples, as described in the text.

$m=(2573.5 \pm 1.7) \mathrm{MeV} / c^{2}$ and $\Gamma=\left(15_{-4}^{+5}\right) \mathrm{MeV}[12]$. Its spin-parity has not been measured but its natural width and decay properties are consistent with a $J^{P}=2^{+}$state [12]. If it is indeed a spin-2 resonance, it cannot be obtained with a $W$-mediated tree diagram but might still be reached through final state interactions.

The allowed decay modes of the $D_{s J}^{+}(2573)$ are $D K$ and $D^{*} K$, both proceeding through a D-wave. Because of the limited phase space, the latter is highly suppressed [17]. In this analysis, a search is made for the $D_{s J}^{+}(2573)$ in the decay mode $D^{0} K^{+}$, in the four channels $B^{0} \rightarrow$ $D^{-} D^{0} K^{+}, D^{*-} D^{0} K^{+}, \bar{D}^{0} D^{0} K^{+}$, and $\bar{D}^{* 0} D^{0} K^{+}$.

The method developed for the $D_{s 1}^{+}(2536)$ study is applied. Figure 5 (b) shows the $\Delta m=m\left(D^{0} K^{+}\right)-m\left(D^{0}\right)$ distribution for the events reconstructed in all four $B \rightarrow$ $\bar{D}^{(*)} D^{0} K^{+}$decay modes. The mean value and the standard deviation of the Gaussian component of the fit function are fixed, respectively, to $\Delta m=708 \mathrm{MeV} / c^{2}$ and $\sigma_{\Delta m}=6 \mathrm{MeV} / c^{2}$, which are the values derived from a large inclusive $D_{s J}^{+}(2573) \rightarrow D^{0} K^{+}$data sample. The fitted yield of $D_{s 1}^{+}(2573) \rightarrow D^{0} K^{+}$decays is $13 \pm 9$ events out of $604 \pm 54 B \rightarrow \bar{D}^{(*)} D^{0} K^{+}$events.

Defining the signal region $687<\Delta m<729 \mathrm{MeV} / c^{2}$,
$90 \%$ C.L. upper limits on the contribution of $D_{s J}^{+}(2573)$ to $B \rightarrow \bar{D}^{(*)} D^{(*)} K$ decays are set for each of the four individual decay modes (Fig. 6(b)). The number of events observed in the signal box, the number of background events expected from the fits, and the resulting limits are given in Table V. $R_{2573}$ is defined here as

$$
R_{2573}=\frac{n\left(B \rightarrow \bar{D}^{(*)} D_{s J}^{+}(2573)\right) \times \mathcal{B}\left(D_{s J}^{+}(2573) \rightarrow D^{0} K^{+}\right)}{n\left(B \rightarrow \bar{D}^{(*)} D^{0} K^{+}\right)} .
$$

TABLE V: $D_{s J}^{+}(2573) \rightarrow D^{0} K^{+}$contributions to $B \rightarrow$ $\bar{D}^{(*)} D^{0} K^{+}$decays.

\begin{tabular}{|l|c|c|c|}
\hline$B$ decay mode & $\begin{array}{c}\text { Total yield } \\
\text { in } D_{s J}^{+}(2573) \\
\text { signal region }\end{array}$ & $\begin{array}{c}\text { Estimated } \\
\text { background }\end{array}$ & $\begin{array}{c}R_{2573} \\
\text { 90\% C.L. } \\
\text { upper limit }\end{array}$ \\
\hline$D^{-} D^{0} K^{+}$ & 25 & $26 \pm 3$ & $6.3 \%$ \\
$D^{*-} D^{0} K^{+}$ & 41 & $42 \pm 3$ & $5.0 \%$ \\
$\bar{D}^{0} D^{0} K^{+}$ & 38 & $29 \pm 3$ & $12.2 \%$ \\
$\bar{D}^{* 0} D^{0} K^{+}$ & 37 & $30 \pm 3$ & $12.3 \%$ \\
\hline
\end{tabular}




\section{Dalitz-plot analysis of the decay $B^{0} \rightarrow D^{*-} D^{* 0} K^{+}$}

As suggested in Ref. [20], the study of decays $B \rightarrow$ $\bar{D}^{(*)} D^{(*)} K$ could be used to search for evidence of the yet undiscovered broad $j_{q}=1 / 2 D_{s J}$ states, if the decays $D_{s J} \rightarrow D^{(*)} K$ are allowed by the available phase space. The decay mode $B^{0} \rightarrow D^{*-} D^{* 0} K^{+}$, which has the largest number of reconstructed events and also has the largest purity, is used for this search. The results are shown in Fig. 7. The upper right plot (Fig. 7 (b)) is the Dalitz plot $m^{2}\left(D^{* 0} K^{+}\right)$vs. $m^{2}\left(D^{*-} D^{* 0}\right)$ expected for three-body $B^{0} \rightarrow D^{*-} D^{* 0} K^{+}$decays generated with a phase space model. The Dalitz plot $m^{2}\left(D^{* 0} K^{+}\right)$ vs. $m^{2}\left(D^{*-} D^{* 0}\right)$ for data events in the signal region $5.27<m_{\mathrm{ES}}\left(D^{*-} D^{* 0} K^{+}\right)<5.29 \mathrm{GeV} / c^{2}$ (Fig. 7 (a)) is shown in Fig. 7 (c). The next three plots (Figs. 7 (d), 7 (e), 7 (f)) show the projections $m\left(D^{* 0} K^{+}\right), m\left(D^{*-} K^{+}\right)$, and $m\left(D^{*-} D^{* 0}\right)$ for the same events. The hatched histograms show the contribution expected from the combinatorial background; their shapes are derived from the events with $m_{\mathrm{ES}}\left(D^{*-} D^{* 0} K^{+}\right)<5.26 \mathrm{GeV} / c^{2}$. The open histograms show the contribution expected for threebody $B^{0} \rightarrow D^{*-} D^{* 0} K^{+}$decays generated with a phasespace model. The density of events in the lower region of the Dalitz plot (i.e., for small values of $m\left(D^{* 0} K^{+}\right)$ and large values of $m\left(D^{*-} D^{* 0}\right)$ ) is significantly larger in the data (Fig. 7 (c)) than in the simulation with no resonance ( Fig. 7 (b)). It could be interpreted as the presence of a broad resonance decaying to $D^{* 0} K^{+}$, like the $J^{P}=1^{+}, j_{q}=1 / 2$ state predicted by Heavy Quark Symmetry models [15-17]. However, more events are necessary to confirm this hypothesis and to estimate the resonance properties such as mass and width.

As previously discussed (Sec. VI), the hypothetical presence of broad resonances in the decay chain is accounted for by a $5 \%$ relative systematic error on all the $B \rightarrow \bar{D}^{(*)} D^{(*)} K$ branching fraction measurements described in this paper.

\section{CONCLUSIONS}

A measurement of the branching fractions for the 22 $B \rightarrow \bar{D}^{(*)} D^{(*)} K$ modes is given in Table I. For the decay modes for which $S / \sqrt{B}$ is smaller than 4 , a $90 \%$ C.L. upper limit is also derived (here, $B$ is the sum of the combinatorial background and the cross-feed background from other $\bar{D}^{(*)} D^{(*)} K$ modes and $S=N-B$, where $N$ is the total yield in the signal region). This is the first complete measurement of all possible $B \rightarrow \bar{D}^{(*)} D^{(*)} K$ channels. The measured branching fractions are in good agreement with earlier measurements made with smaller data sets for some of these modes [5-8].

The existence of the decays $B^{0} \rightarrow D^{*-} D^{+} K_{S}^{0}$ and $B^{0} \rightarrow D^{*-} D^{*+} K_{S}^{0}$, which are an admixture of $C P$ even and $C P$ odd eigenstates, has been demonstrated. These decay modes could be used in the future, with larger event samples, to also determine $\sin 2 \beta$ and $\cos 2 \beta[21-$ 23]. A significant signal for the color suppressed decay mode $B^{+} \rightarrow D^{*-} D^{+} K^{+}$has also been observed.

One of the motivations of this analysis is to understand whether decays $B \rightarrow \bar{D}^{(*)} D^{(*)} K$ can explain the wrongsign $D$-meson rates in $B$ decays and reconcile the total $b \rightarrow c \bar{c} s$ rate with the predictions of Ref. [3]. After summing over all submodes, the branching fractions of the $B^{0}$ and of the $B^{+}$to $\bar{D}^{(*)} D^{(*)} K$ are found to be

$$
\begin{gathered}
\mathcal{B}\left(B^{0} \rightarrow \bar{D}^{(*)} D^{(*)} K\right)=(4.3 \pm 0.3(\text { stat }) \pm 0.6(\text { syst })) \% \\
\mathcal{B}\left(B^{+} \rightarrow \bar{D}^{(*)} D^{(*)} K\right)=(3.5 \pm 0.3(\text { stat }) \pm 0.5(\text { syst })) \%
\end{gathered}
$$

This study shows that a significant fraction of the transitions $b \rightarrow c \bar{c} s$ proceed through the decays $B \rightarrow$ $\bar{D}^{(*)} D^{(*)} K$. These decay modes account for about one half of the wrong-sign $D$ production rate in $B$ decays, $\mathcal{B}(B \rightarrow D X)=(7.9 \pm 2.2) \%[4]$; however, because of the large statistical error on the latter measurement, it is not yet clear whether they saturate it.

A search for resonant substructures shows that the $D_{s 1}^{+}(2536)$ contribution to $B \rightarrow \bar{D}^{(*)} D^{* 0} K^{+}$decays is small. No evidence for a $D_{s J}^{+}(2573)$ contribution to $B \rightarrow \bar{D}^{(*)} D^{0} K^{+}$decays is found. Finally, a simple Dalitz-plot analysis of the decays $B^{0} \rightarrow D^{*-} D^{* 0} K^{+}$ shows that the three-body phase-space decay model does not give a satisfactory description of these decays.

\section{ACKNOWLEDGMENTS}

We are grateful for the extraordinary contributions of our PEP-II colleagues in achieving the excellent luminosity and machine conditions that have made this work possible. The success of this project also relies critically on the expertise and dedication of the computing organizations that support BABAR. The collaborating institutions wish to thank SLAC for its support and the kind hospitality extended to them. This work is supported by the US Department of Energy and National Science Foundation, the Natural Sciences and Engineering Research Council (Canada), Institute of High Energy Physics (China), the Commissariat à l'Energie Atomique and Institut National de Physique Nucléaire et de Physique des Particules (France), the Bundesministerium für Bildung und Forschung and Deutsche Forschungsgemeinschaft (Germany), the Istituto Nazionale di Fisica Nucleare (Italy), the Foundation for Fundamental Research on Matter (The Netherlands), the Research Council of Norway, the Ministry of Science and Technology of the Russian Federation, and the Particle Physics and Astronomy Research Council (United Kingdom). Individuals have received support from the A. P. Sloan Foundation, the Research Corporation, and the Alexander von Humboldt Foundation. 

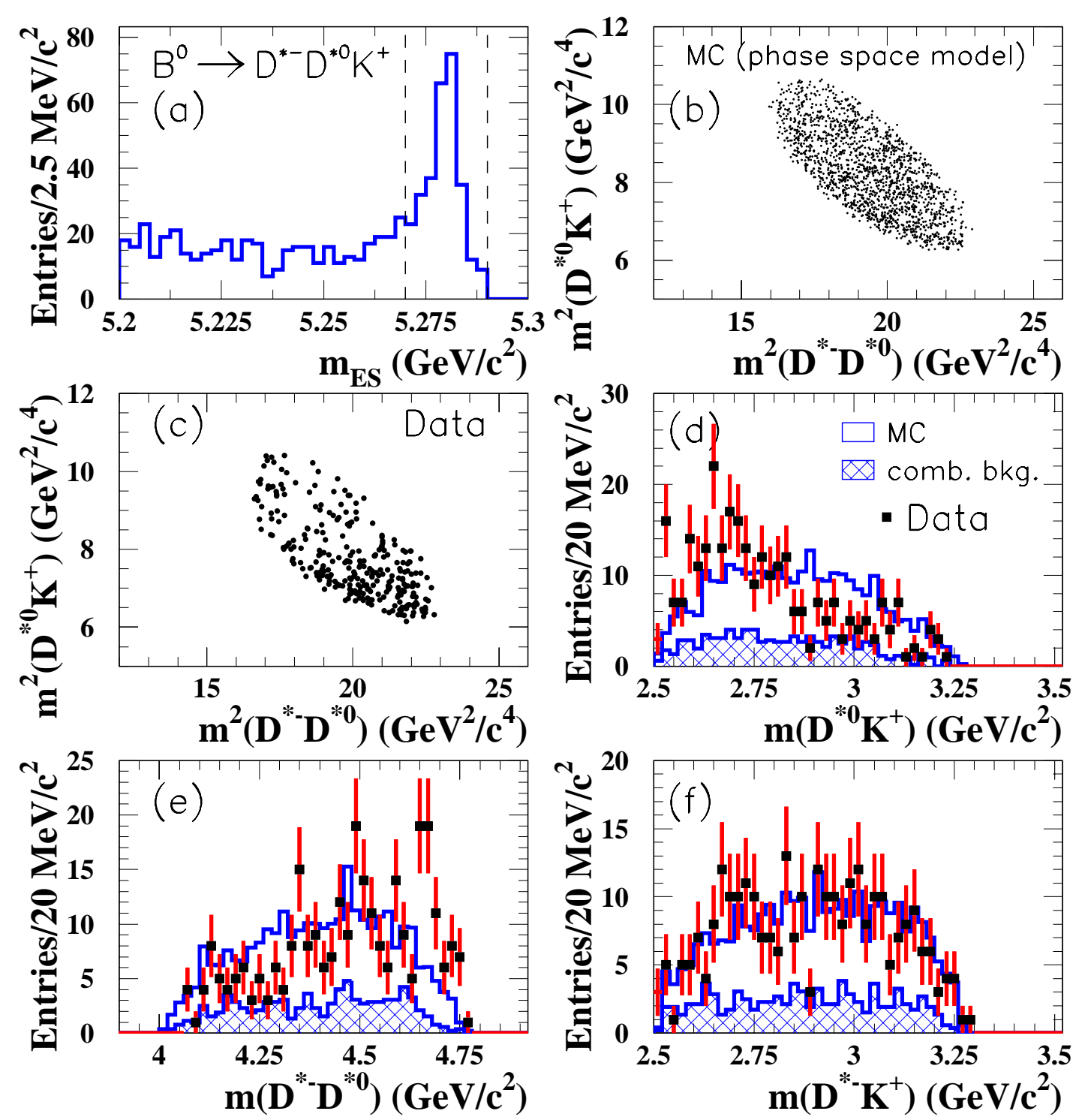

FIG. 7: Dalitz plots and projections for the decay $B^{0} \rightarrow D^{*-} D^{* 0} K^{+}$. The content of the different plots is discussed in the text.

[1] T. Browder, Proceedings of the 1996 Warsaw ICHEP Conference, edited by Z. Ajduk and A.K. Wroblewski (World Scientific, 1997), p. 1139.

[2] I. I. Bigi, B. Blok, M. Shifman, and A. Vainshtein, Phys. Lett. B 323, 408 (1994).

[3] G. Buchalla, I. Dunietz, and H. Yamamoto, Phys. Lett. B 364, 188 (1995).

[4] CLEO Collaboration, T. E. Coan et al., Phys. Rev. Lett. 80, 1150 (1998).
[5] CLEO Collaboration, CLEO CONF 97-26, EPS97 337 (1997).

[6] ALEPH Collaboration, R. Barate et al., Eur. Phys. Jour. C 4, 387 (1998).

[7] BABAR Collaboration, B. Aubert et al., SLAC-PUB8924, hep-ex/0107056, presented at the 20th International Symposium on Lepton and Photon Interactions at High Energies, Rome, Italy (2001).

[8] Belle Collaboration, K. Abe et al., BELLE-CONF-0104, 
presented at the 20th International Symposium on Lepton and Photon Interactions at High Energies, Rome, Italy (2001).

[9] BABAR Collaboration, B. Aubert et al., Nucl. Instr. Meth. A 479, 1 (2002).

[10] G.C. Fox and S. Wolfram, Nucl. Phys. B 149, 413 (1979).

[11] ARGUS Collaboration, H. Albrecht et al., Phys. Lett. B 241, 278 (1990).

[12] Particle Data Group, K. Hagiwara et al., Phys. Rev. D 66, 010001 (2002).

[13] The Geant4 Collaboration, CERN-IT-2002-003, submitted to Nucl. Instrum. Methods (2002).

[14] CLEO Collaboration, M. Procario et al., Phys. Rev. Lett. 70, 1207 (1993).

[15] J. L. Rosner, Comm. Nucl. Part. Phys. 16, 109 (1986) and references therein.

[16] N. Isgur and M. B. Wise, Phys. Rev. Lett. 66, 1130
(1991).

[17] S. Godfrey and R. Kokoski, Phys. Rev. D 43, 1679 (1991).

[18] CLEO Collaboration, M. Bishai et al., Phys. Rev. D 57, 3847 (1998).

[19] BABAR Collaboration, B. Aubert et al., SLAC-PUB-9711, hep-ex/0304021, submitted to Phys. Rev. Lett. .

[20] P. Colangelo and F. De Fazio, Phys. Lett. B 532, 193 (2002).

[21] J. Charles, A. Le Yaouanc, L. Oliver, O. Pene, and J. C. Raynal, Phys. Lett. B 425, 375 (1998) [Erratumibid. B 433, 441 (1998)].

[22] P. Colangelo, F. De Fazio, G. Nardulli, N. Paver and Riazuddin, Phys. Rev. D 60, 033002 (1999).

[23] T. E. Browder, A. Datta, P. J. O'Donnell and S. Pakvasa, Phys. Rev. D 61, 054009 (2000). 